JOURNAL OF THE

AMERICAN MATHEMATICAL SOCIETY

Volume 21, Number 1, January 2008, Pages 137-169

S 0894-0347(07)00557-7

Article electronically published on February 20, 2007

\title{
THE CONFORMALLY INVARIANT MEASURE ON SELF-AVOIDING LOOPS
}

\author{
WENDELIN WERNER
}

\section{INTRODUCTION}

The aim of the present paper is to construct and describe a natural measure on the set of self-avoiding loops in the plane and on any Riemann surface. By a self-avoiding loop on a surface $S$, we mean a continuous injective map from the unit circle into $S$ modulo monotone reparametrizations (i.e. we look only at the trace of the loop and forget about its parametrization). We will construct a measure that possess some strong conformal invariance properties, and we shall see that this measure is the only one with these properties.

Let us first describe this strong conformal invariance property, phrased in terms of a measure on the set of self-avoiding loops in the plane: We say that such a measure $\mu$ satisfies conformal restriction if for any two conformally equivalent domains $D$ and $D^{\prime}$ (i.e. such that there exists a conformal map from $D$ onto $D^{\prime}$ ) in the plane, the image of the measure $\mu$ restricted to the set of loops that stay in $D$, via any conformal map $\Phi$ from $D$ onto $D^{\prime}$, is exactly the measure $\mu$ restricted to the set of loops that stay in $D^{\prime}$.

Note that this condition implies in particular that the measure $\mu$ is translationinvariant, scale-invariant (and therefore that it has infinite total mass provided that $\mu \neq 0$ ), and that if $\mu$ satisfies conformal restriction, then so does $c \mu$ for any positive constant $c$. We are going to say that a measure on the set of self-avoiding loops is non-trivial if for some $0<\delta<\Delta<\infty$, the mass of the set of loops of diameter at least $\delta$ and that stay in some disc of radius $\Delta$ is neither 0 nor infinite.

We shall prove the following result:

Theorem 1. Up to multiplication by a positive constant, there exists a unique nontrivial measure on the set of self-avoiding loops in the plane that satisfies conformal restriction.

Before giving some motivation, let us now describe the counterpart of this result in term of measures on loops on Riemann surfaces. Suppose that for each Riemann surface $S$ (we do not require $S$ to be closed), we are given a measure $\mu_{S}$ on the set of self-avoiding loops on $S$. We say that the family of measures $\left(\mu_{S}\right)$ satisfies conformal restriction if the following two conditions hold:

- For any conformal map $\Phi$ from one surface $S$ onto another surface $S^{\prime}$, $\Phi \circ \mu_{S}=\mu_{S^{\prime}}$

Received by the editors December 17, 2005.

2000 Mathematics Subject Classification. Primary 60D05; Secondary 82B41, 82B43, 30C99, $60 \mathrm{~J} 65$

This work was supported by the Institut Universitaire de France.

(C)2007 American Mathematical Society 137 


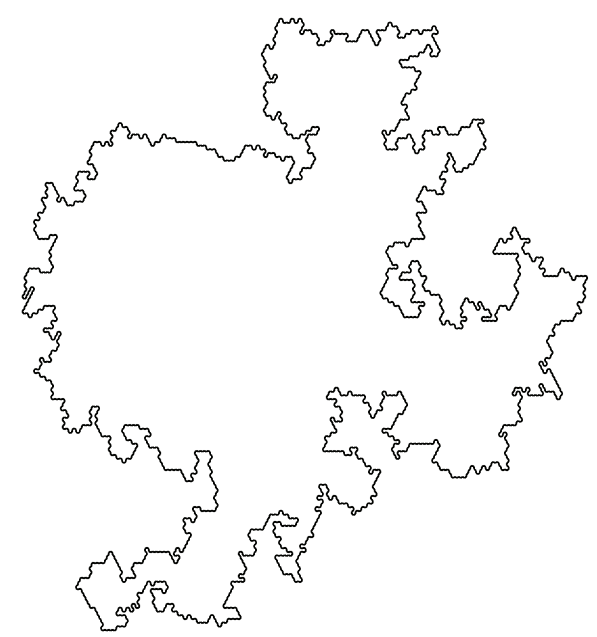

Figure 1. A self-avoiding loop (sketch)

- For any $S$ and any $S^{\prime} \subset S, \mu_{S^{\prime}}$ is equal to the measure $\mu_{S}$ restricted to those loops that stay in $S^{\prime}$.

Suppose that the family $\left(\mu_{S}\right)$ satisfies conformal restriction. It is then clear that the measure $\mu=\mu_{\mathbb{C}}$ in this family satisfies conformal restriction in the previous sense. It is also easy to see that for any Riemann surface $S$, one can define a countable family of annular regions (i.e. conformally equivalent to a planar annular region) such that any self-avoiding loop in $S$ is contained in at least one of these regions. Hence, the knowledge of the $\mu_{D}$ 's for all annular regions $D$ determines the entire family $\left(\mu_{S}\right)$. One consequence is that the entire family $\left(\mu_{S}\right)$ is described by the measure $\mu=\mu_{\mathbb{C}}$. This will lead to

Theorem 2. Up to multiplication by a positive constant, there exists a unique family of such measures $\left(\mu_{S}\right)$ that satisfies conformal restriction.

The proof of these results will consist of two different parts. First, one shows that there exists at most one measure $\mu$ that satisfies conformal restriction, and then one constructs explicitly a measure $\mu$ that possesses this property. As we shall see, the first step is not very difficult and requires no prerequisite. On the other hand, the existence part of the proof of the theorem is non-trivial. Recall that we are not restricting ourselves to simply connected domains $D$; for instance, the measure $\mu$ will be invariant under the inversion $z \rightarrow 1 / z$ from $\mathbb{C} \backslash\{0\}$ onto itself.

Let us now list some other properties of the measures described in Theorems 1 and 2 that we shall derive in this paper:

- For any given simply connected domain $D \subset \mathbb{C}$, for any simply connected $D^{\prime} \subset D$, and $z \in D^{\prime}$, consider the conformal map $\Phi$ from $D^{\prime}$ onto $D$ that fixes $z$ and that has a positive derivative at $z$. Then

$$
\mu_{D}\left(\left\{\gamma: \gamma \text { disconnects } z \text { from } \partial D \text { and } \gamma \not \subset D^{\prime}\right\}\right)=c \log \Phi^{\prime}(z)
$$

for some $c$. This formula (for fixed $D$ and letting $z$ and $D^{\prime}$ vary) characterizes the measure $\mu_{D}$ fully (and therefore $\mu$ too because of its scaleinvariance). 
- The measure $\mu$ can be viewed as the measure on outer boundaries of planar Brownian loops in the plane, where these Brownian loops are defined under the "Brownian loop-measure" introduced in 24]. This is roughly speaking a scale-invariant and translation-invariant measure on (unrooted) Brownian loops in the plane.

- For each Riemann surface $S$ (and in particular for $S=\mathbb{C}$ ), the measure $\mu_{S}$ is supported on the set of loops with Hausdorff dimension 4/3 (see Figure1). This will follow from the construction of $\mu$ via outer boundaries of Brownian loops that we have just mentioned (recall that it has been proved - see 18, 19 - that the dimension of outer boundaries of two-dimensional Brownian motions is $4 / 3$ ).

- One can also view the measures $\mu_{S}$ as measures on "Schramm-Loewner Evolutions loops" with parameter $8 / 3$. This is related to the special properties of SLE with that parameter (e.g. 21]). It will be an instrumental fact in the proof of Theorems 1 and 2 .

- One can also interpret the measure $\mu$ as the scaling limit of the measure on critical percolation cluster outer perimeters. In particular, this will show that the shapes of percolation cluster outer perimeters in the scaling limit are exactly the same (in law) as that of Brownian loops.

- The $\mu$ mass of the set of loops that stay and go "around" the annulus $\{z:|z| \in(1, \exp (\rho))\}$ decays like a constant times $\exp \left\{-5 \pi^{2} / 4 \rho\right\}$ when $\rho \rightarrow 0+$. This estimate also holds for other annular regions by conformal invariance.

This last estimate can be related to the conjecture that the measures $\mu_{S}$ are the scaling limits of simple measures on self-avoiding polygons on fine-mesh discrete approximations of the surface $S$ (see Section 7.1 and [20]). Roughly speaking, $c \mu$ for some $c$ should be the fine-mesh limit (i.e. $\delta \rightarrow 0$ ) of the measure that assigns a mass $\lambda^{-n(l)}$ to each self-avoiding loop $l$ on the grid $\delta \mathbb{Z}^{2}$, where $n(l)$ denotes the number of steps of the loop $l$ and $\lambda$ is the connectivity constant of the lattice $\mathbb{Z}^{2}$.

The description of measures $\mu$ in terms of Brownian outer boundaries or percolation outer perimeters in the scaling limit, combined with the properties of the measures $\mu$ (such as for instance the invariance under inversion $z \mapsto 1 / z$ ) has in turn rather surprising consequences for Brownian loops and shapes of percolation clusters. In particular, it will show that the shapes of "outer" and "inner" boundaries have exactly the same law.

The paper will be structured as follows: in the next section, which can be viewed as the second part of the introduction, we make some further general comments and recall a few general facts and ideas from the papers 24 and 21. In Section 3, we show uniqueness of the measure $\mu$ satisfying a weaker form of conformal restriction (namely that the restrictions of $\mu$ to simply connected sets are all conformally equivalent). This implies in particular the uniqueness part of Theorem 1. In the subsequent section, we will then show how to construct a measure $\mu$ satisfying this weak conformal restriction property using the Brownian loop-measures (to each Brownian loop we associate its outer boundary which is a self-avoiding loop). We will then focus on the restriction of this measure to annular regions. In particular, using $\mathrm{SLE}_{8 / 3}$ considerations, we shall prove that for any two conformally equivalent annular regions in the plane, the restriction of $\mu$ to the second one is equal to the conformal image of the restriction of $\mu$ to the first one. This will in turn imply 
Theorem 1 and show that it is possible to define $\mu_{S}$ for any surface in such a way that the obtained family of measures satisfies conformal restriction (i.e. that Theorem 2 holds too). In the final two sections, we will study asymptotics of the mass of loops that go around thin annuli (motivated by the discrete self-avoiding walks problems) and the relation to critical percolation.

\section{FURTHER MOTIVATION AND BACKGROUND}

This paper builds on ideas of the joint paper with Greg Lawler and Oded Schramm on conformal restriction in the chordal case [21] and uses also the Brownian loop-measure introduced in joint work with Greg Lawler [24]. A survey of the results concerning SLE with parameter $8 / 3$ and Brownian measures (on excursions and loops) that we will use can for instance be found in the first four sections of [40. Let us however briefly recall some aspects of the two papers [24, 21] now, in order to put our results into perspective:

Self-avoiding loops and Brownian loops. Because of its conformal invariance, planar Brownian motion can be a useful tool to interpret and prove results in complex analysis (e.g. 2]) even though many questions in this field were already settled long before Brownian motion was properly defined. Usually, by conformal invariance of planar Brownian motion one means that the conformal image of a Brownian motion started at a given point in a given domain is a (time-changed) Brownian motion in the image domain started at the image of the starting point (this had been first observed by Paul Lévy in the 1950's and it can be viewed as a direct consequence of Itô's formula; see e.g. [26]). If one does however not prescribe any starting point on a Riemann surface and still wants to state a conformal invariance property for measures on Brownian paths, it is natural to look for measures on Brownian loops. In 24, we defined a measure on Brownian loops in the complex plane (and subsets of the complex plane) that is indeed conformally invariant. More precisely, it satisfies a property similar to the conformal restriction that we described before (but of course, Brownian loops are not simple loops): The image under a conformal map $\Phi$ of the measure on Brownian loops restricted to those that stay in a domain $D \subset \mathbb{C}$ is exactly the measure on Brownian loops restricted to those loops that stay in $\Phi(D)$. As we shall see later in this paper, it is easy to generalize the definition of the Brownian loop-measure to general Riemann surfaces and to show that this conformal restriction property still holds.

In the case of hyperbolic surfaces (where Green's functions are finite) such for instance as the bounded subsets of $\mathbb{C}$, quantities like the mass of Brownian loops that intersect two disjoint sets turn out to be rather natural conformal invariants that are for instance related to Schwarzian derivatives (see e.g. 21) and they show up under various guises in conformal field theory. Furthermore [24, 38], it is natural to construct Poissonized samples of these Brownian loop-measures (that we call the Brownian loop-soups in 24]) and to study the geometry of the obtained sets [38. This gives a way to construct the so-called conformal loop ensembles (CLE) that are conjectured to be the scaling limits of various critical two-dimensional models from statistical physics; see [34, 41].

The Brownian loop-measure on compact surfaces (for which Green's functions are infinite), such as for instance the Riemann sphere, is still easy to define but not so easy to work with. For instance, the mass of the set of loops that intersect two 
disjoint open sets is infinite because of the too many long loops (due to the recurrence of the Brownian motion). This implies in particular that the corresponding Brownian loop-soups become rather uninteresting, because there always exists just one dense cluster of loops. However, it should be possible to define the CLEs and conformal field theories on such surfaces in order to describe scaling limits of lattice models.

We shall see that the measure on self-avoiding loops $\mu_{S}$ that we are defining is in a way better-suited to higher-genus surfaces and compact surfaces than the Brownian loop-measure. For simply connected planar domains, the self-avoiding loop-measure $\mu$ is very directly related to the Brownian loop-measure (it is the measure on "outer boundaries of the Brownian loops") and therefore also to Schwarzian derivatives, etc. Its Poissonian samples also define the same CLEs as the Brownian loop-measure does. But these nice properties will still hold for any Riemann surface. For instance, it allows us to construct directly loop-clusters for a loop-soup in the entire plane. In this setup, all the nice properties of the obtained cluster boundaries are just consequences of the conformal restriction property of the family $\left(\mu_{S}\right)$.

Chordal restriction. Let us now briefly recall some aspects of conformal restriction in the chordal case, as studied in [21. There, we focused on probability measures on self-avoiding curves that join two prescribed boundary points of simply connected domains (we will only discuss simply connected domains in this paragraph). The law of such a curve was therefore characterized by the domain $D$ and also by the two endpoints $A$ and $B$ of the path. We showed that there exists a unique family $\left(P_{D, A, B}\right)$ of probability measures on that set of self-avoiding curves that satisfies conformal invariance (i.e. $\Phi \circ P_{D, A, B}=P_{\Phi(D), \Phi(A), \Phi(B)}$ ) and restriction in the sense that the law of the curve in $D$, conditioned to stay in $D^{\prime}$, was equal to the law in the smaller domain with the boundary points $A$ and $B$. The constraint on $D^{\prime}$ is that $A$ and $B$ are also boundary points of $D^{\prime}$ and for instance that $D \backslash D^{\prime}$ is at a positive distance from $A$ and $B$ (to ensure that the curve stays in $D^{\prime}$ with positive probability so that the conditioning makes sense). Furthermore (see 21] for more details), we showed that

$$
P_{D, A, B}\left(\gamma \subset D^{\prime}\right)=\left(\Phi^{\prime}(A) \Phi^{\prime}(B)\right)^{5 / 8}
$$

when $\Phi$ is a conformal map from $D^{\prime} \subset D$ back onto $D$ that preserves the two boundary points $A$ and $B$.

One can then try to define a family of measures $\mu_{D, A, B}$ on such curves in such a way that $\mu_{D, A, B}$ restricted to those curves that stay in $D^{\prime}$ is exactly equal to $\mu_{D^{\prime}, A, B}$. In other words, we allow the measure to have a total mass different to 1 , but we require that the restriction property hold with no renormalizing term (for the family $P_{D, A, B}$, there is such a term due to the conditioning). Let us add the technical condition that we restrict the definition of $\mu_{D, A, B}$ to those sets $D$ and points $A, B$, such that the boundary of $D$ is smooth in the neighborhood of $A$ and $B$. Then, we simply define

$$
\mu_{D, A, B}=\Phi^{\prime}(A)^{-5 / 8} \Phi^{\prime}(B)^{-5 / 8} P_{D, A, B},
$$

where $\Phi$ is a conformal map from some fixed reference domain (say $\mathbb{H}, 0, \infty$ ) onto $D, A, B$. The family of measures $\mu_{D, A, B}$ is then defined modulo a multiplicative constant (corresponding to the choice of the reference domain) and can be shown to 
be the unique one (on the set of simple paths from one boundary point to another of smooth simply connected domains) satisfying this restriction property.

It was very natural to study this chordal restriction property in light of the definition and properties of the chordal Schramm-Loewner evolutions that are precisely random paths joining one boundary point of a domain to another. In fact, the probability $P_{D, A, B}$ is exactly the law of the SLE with parameter $8 / 3$ in $D$ from $A$ to $B$. It also turned out (see e.g. [13, 3]) that this chordal restriction property of SLE with parameter $8 / 3$ had interpretations in terms of boundary conformal field theory and that it yields a simple definition of the $\mathrm{SLE}_{8 / 3}$ in non-simply connected domains. Also, a by-product of the results of [21] was the fact that the Brownian outer boundary looked "locally" like that of a critical percolation boundary, and also like an $\mathrm{SLE}_{8 / 3}$, because of global identities in law between outer boundaries of the union of five Brownian excursions and that of the union of eight $\mathrm{SLE}_{8 / 3}$ 's. This showed that "locally" the inside boundaries and the outside boundaries of the Brownian loops and of percolation clusters looked like an $\mathrm{SLE}_{8 / 3}$.

So, we see that part of the present paper is really a continuation of some of the ideas developed in 21. In a way, just as for the Brownian loops compared to Brownian motions, the measure on self-avoiding loops $\mu$ in the plane is invariant under a wider class of transformations than the chordal measures, because no marked points are prescribed. This will make it well-suited to general Riemann surfaces and to the description and construction of conformally invariant quantities and objects.

Further motivation. It is also worth emphasizing that the conformal restriction property enables us to understand the behavior of these measures when one distorts locally the Riemann surface (for instance, one cuts out a small disc and glues some other structure), so that $\mu$ provides conformally invariant information that behaves nicely under perturbation of the conformal structure. We believe that it is a natural and hopefully fruitful way to describe useful conformal invariant quantities in this setup [42].

Also, one can note (see e.g. [30]) that the space of shapes of self-avoiding loops (as we shall define it later in the paper) can be realized in many different ways in particular involving the Lie algebra $\operatorname{Vect}\left(S^{1}\right)$ and that it has been the subject of many investigations (see e.g. 27]). We hope to clarify the link with the present paper in [42]. Measures on loops should also be the natural setup for considerations related to the geometric-theoretical aspects of SLE as looked into in [12, 11, 15].

\section{Simply connected Case: Uniqueness}

This section requires no prerequisite (i.e. no knowledge on Brownian loops nor on SLE).

Let us settle a few technical details: Throughout the paper, when we talk about self-avoiding loops, we will not care about their time-parametrizations. In other words, we identify two self-avoiding loops as soon as their traces are the same. This means that two injective continuous maps $\gamma^{1}$ and $\gamma^{2}$ from the unit circle $S^{1}$ into some domain define the same loop if there exists a continuous monotone bijection $w$ from $S^{1}$ onto $S^{1}$ such that $\gamma^{1}=\gamma^{2} \circ w$. Note that with this definition, a path of zero length (i.e. "a point") is not a self-avoiding loop.

This set of self-avoiding loops can be endowed with various natural metrics. We will not discuss this here, but we have at least to specify precisely what $\sigma$-field we will implicitly refer to when we define measures on this set. Throughout the 
paper, we will say that a bounded open set $A$ is an annular region (not to be confused with exact annuli) in the plane if it is conformally equivalent to some annulus $\{z: 1<|z|<R\}$. In other words, $A$ is a bounded open set and $\mathbb{C} \backslash A$ has two connected components (that are not singletons). The bounded connected component of $\mathbb{C} \backslash A$ will be called the "hole" of $A$. For each such annular region $A$, define the set $\mathcal{U}_{A}$ of self-avoiding loops that stay in $A$ and that have a non-zero index around the hole of $A$ (or in other words, that disconnects the two connected components of $\partial A$ ). We will denote by $\mathcal{G}$ the $\sigma$-field generated by this family. This is the $\sigma$-field that we will work with (we leave it to the interested reader to check the relation between $\mathcal{G}$ and the Borel $\sigma$-field induced by the Hausdorff distance on the set of self-avoiding loops).

We will also use the countable family $\mathcal{A}$ of annular regions $A$ such that the inner and the outer boundaries of $A$ consist of finite loops that are drawn on the lattice $2^{-p} \mathbb{Z}^{2}$ for some $p \geq 1$. Clearly, since any annular region can be seen as the increasing limit of such dyadic annular regions, the family $\left(\mathcal{U}_{A}, A \in \mathcal{A}\right)$ generates $\mathcal{G}$ too. The sets of self-avoiding loops that we shall consider can be easily shown to be in $\mathcal{G}$ (for instance because they are countable unions of $\mathcal{U}_{A}$ 's).

When $\mu$ is a measure supported on the set of self-avoiding loops in the complex plane, then, for each simply connected domain $D$ (unless otherwise stated, domains are open sets), we simply define $\mu_{D}$ to be the measure $\mu$ restricted to the set of loops that stay in $D$. Throughout this paper, by a slight abuse of notation, we will write $\mu_{D}=\mu 1_{\{\gamma \subset D\}}$ (instead of $\mu_{D}(\cdot)=\mu(\cdot \cap\{\gamma \subset D\})$ ).

Recall that we say that a measure on the set of self-avoiding loops is non-trivial if for some $0<\delta<\Delta<\infty$, the mass of the set of loops of diameter at least $\delta$ that are contained in some disc of radius $\Delta$ is neither zero nor infinity.

We will also use a weaker form of conformal restriction in this paper: We will say that a measure $\mu$ on the set of self-avoiding loops in the plane satisfies weak conformal restriction if for any two simply connected domains $D, D^{\prime}$ and any conformal map $\Phi$ from $D$ onto $D^{\prime}$,

$$
\Phi \circ \mu_{D}=\mu_{D^{\prime}}
$$

The difference with the (strong) conformal restriction is that here the domains $D$ and $D^{\prime}$ are simply connected.

We can now state the main result of this section:

Proposition 3. Up to multiplication by a positive constant, there exists at most one non-trivial measure $\mu$ (on the set of self-avoiding loops) satisfying weak conformal restriction.

Furthermore, for any simply connected sets $\tilde{D} \subset D$ and any $z \in \tilde{D}$,

$$
\mu(\{\gamma: \gamma \subset D, \gamma \not \subset \tilde{D}, \gamma \text { disconnects } z \text { from } \partial D\})=c \log \Phi^{\prime}(z),
$$

where $c$ is a positive constant (depending on $\mu$ only) and $\Phi$ denotes the conformal map from $\tilde{D}$ onto $D$ such that $\Phi(z)=z$ and $\Phi^{\prime}(z)$ is a positive real.

This result does not say anything about whether this measure exists.

We will also prove the following closely related lemma. It is a similar result for measures on loops that surround a given point. Suppose that $\nu$ is a non-trivial measure supported on the set of self-avoiding loops in the complex plane that disconnect 0 from infinity. For any simply connected domain $D$ containing the origin, we define again $\nu_{D}=\nu 1_{\gamma \subset D}$. 
Lemma 4. Up to multiplication by a positive constant, there exists at most one such measure $\nu$ such that for any simply connected domain $D$ containing the origin and any conformal map $\Phi$ defined on $D$ such that $\Phi(0)=0$, one has $\Phi \circ \nu_{D}=\nu_{\Phi(D)}$. Furthermore, there exists a positive constant c such that for any simply connected subset $U$ of the unit disc $\mathbb{U}$ such that $0 \in U$,

$$
\nu(\{\gamma: \gamma \subset \mathbb{U}, \gamma \not \subset U\})=c \log \left(\Phi^{\prime}(0)\right)
$$

where $\Phi$ is the conformal map from $U$ onto $\mathbb{U}$ such that $\Phi(0)=0$ and $\Phi^{\prime}(0)>0$.

We will first prove the lemma.

Proof of the lemma. Suppose that $\nu$ is a measure supported on the set of selfavoiding loops that surround the origin, such that for any simply connected domain $D$ containing the origin and any conformal map $\Phi: D \rightarrow \Phi(D)$ with $\Phi(0)=0$, one has $\nu_{\Phi(D)}=\Phi \circ \nu_{D}$. Our first goal is to prove that it necessarily satisfies (3.2).

Consider a simply connected subset $U$ of the unit disc that contains the origin. We define the unique conformal map $\varphi=\varphi_{U}$ from $U$ onto $\mathbb{U}$ with $\varphi(0)=0$ and $\varphi^{\prime}(0)>0$. Note that as $U \subset \mathbb{U}, \varphi^{\prime}(0) \geq 1$ (recall for instance that if $Z$ is a planar Brownian motion started from the origin and stopped at its first exit time $T$ of $U$, then $\left.\log \left(\varphi^{\prime}(0)\right)=-E\left(\log \left|Z_{T}\right|\right) \geq 0\right)$.

Note that $\varphi$ describes $U$ fully as $U=\varphi^{-1}(\mathbb{U})$. We now define

$$
A\left(\varphi_{U}\right)=\nu(\{\gamma: \gamma \subset \mathbb{U}, \gamma \not \subset U\}) \text {. }
$$

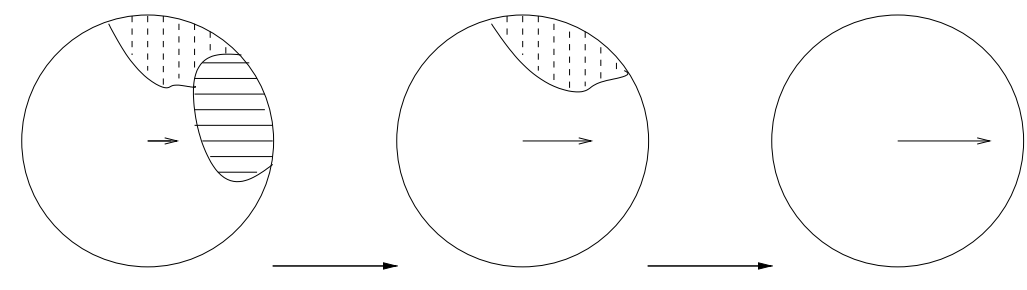

FIGURE 2. $\varphi_{V} \circ \varphi_{U}$

This quantity measures the mass of the set of loops that stay in the unit disc but do exit the smaller domain $U$ (recall also that $\nu$ is supported on the set of loops that surround the origin). Now, under the conditions of the lemma, it is immediate to see that for any simply connected subsets $U$ and $V$ of the unit disc that do contain the origin,

$$
A\left(\varphi_{V} \circ \varphi_{U}\right)=A\left(\varphi_{U}\right)+A\left(\varphi_{V}\right) .
$$

Indeed, $A\left(\varphi_{V} \circ \varphi_{U}\right)$ is the mass of loops that surround the origin, stay in $\mathbb{U}$, but hit $\mathbb{U} \backslash U$ or $\varphi_{U}^{-1}(\mathbb{U} \backslash V)$. Note that these two sets are disjoint. But by definition

$$
\nu(\{\gamma: \gamma \subset \mathbb{U}, \gamma \not \subset U\})=A\left(\varphi_{U}\right)
$$

and

$$
\nu(\{\gamma: \gamma \subset \mathbb{U}, \gamma \not \subset V\})=A\left(\varphi_{V}\right) .
$$

By conformal invariance (via $\varphi_{U}^{-1}$ ), we see that this second quantity is also equal 
to

$$
\nu\left(\left\{\gamma: \gamma \subset U, \gamma \not \subset \varphi_{U}^{-1}(V)\right\}\right) .
$$

Summing (3.4) and (3.5), we get 3.3).

Let us now focus on a simple class of domains $U_{t}$ in $\mathbb{U}$ : For each positive $t$, we let $U_{t}=\mathbb{U} \backslash\left[r_{t}, 1\right)$, where $r_{t}$ is the positive real such that $\varphi_{U_{t}}^{\prime}(0)=e^{t}$ (it is not difficult to work out the explicit expression of $r_{t}$ but we do not need it here). Note that with this definition, the family $\left(\varphi_{U_{t}}\right)_{t \geq 0}$ is a semi-group (i.e. $\varphi_{U_{t}} \circ \varphi_{U_{s}}=\varphi_{U_{s+t}}$ ) because (by symmetry) the composition of these two maps is still a map from some $U_{r}$ onto $\mathbb{U}$, and $r=s+t$ because $\left(\varphi_{U_{t}} \circ \varphi_{U_{s}}\right)^{\prime}(0)=e^{s+t}$. Hence, $t \mapsto A\left(\varphi_{U_{t}}\right)$ is a non-decreasing function from $(0, \infty)$ into $(0, \infty)$, such that

$$
A\left(\varphi_{U_{t+s}}\right)=A\left(\varphi_{U_{t}}\right)+A\left(\varphi_{U_{s}}\right)
$$

This implies that for some positive constant $c$, we have that $A\left(\varphi_{U_{t}}\right)=c t=$ $c \log \varphi_{U_{t}}^{\prime}(0)$ (the constant is positive and finite because $\nu$ is non-trivial). In the sequel, we fix $c$.

For each $\theta \in[0,2 \pi)$ and $t>0$, define the set

$$
U_{t, \theta}=\mathbb{U} \backslash\left[r_{t} e^{i \theta}, e^{i \theta}\right)
$$

Clearly, $\varphi_{U_{t, \theta}}^{\prime}(0)=t$ and because of the invariance of $\nu$ under rotations, we have $A\left(\varphi_{U_{t, \theta}}\right)=A\left(\varphi_{U_{t}}\right)=c t$.

Let $\mathcal{S}$ denote the semi-group of conformal maps generated by the family $\left(\varphi_{U_{t, \theta}}\right.$, $t>0, \theta \in[0,2 \pi)$ ) (i.e. the set of finite compositions of such maps). Because of (3.3), we deduce from the above that for any $\varphi \in \mathcal{S}$,

$$
A(\varphi)=c \log \left(\varphi^{\prime}(0)\right) .
$$

The family $\mathcal{S}$ is "dense" in the class of conformal maps $\varphi_{U}$ from some simply connected subset $U$ onto $\mathbb{U}$ in the following sense (this is a standard fact from the theory developed by Loewner): For any $U$, there exists an increasing family $U_{n}$ in $\mathcal{S}$ such that $\bigcup_{n} U_{n}=U$. Clearly, it follows that $\varphi_{U_{n}}^{\prime}(0) \rightarrow \varphi_{U}^{\prime}(0)$. Furthermore, because a loop is a compact subset of the complex plane, $\gamma$ exits $U$ if and only if it exits all $U_{n}$ 's, so that $A\left(\varphi_{U_{n}}\right) \rightarrow A\left(\varphi_{U}\right)$ as $n \rightarrow \infty$. Combining the above, we get that for any $U$,

$$
A\left(\varphi_{U}\right)=\lim _{n} A\left(\varphi_{U_{n}}\right)=c \lim _{n} \log \varphi_{U_{n}}^{\prime}(0)=c \log \varphi_{U}^{\prime}(0) .
$$

This shows that $\nu$ must indeed satisfy 3.2 .

The lemma will then follow from rather simple measure-theoretical considerations that we now detail. Recall that a finite measure on a measurable space is characterized by its total mass and its value on a subset of the $\sigma$-field that generates the $\sigma$-field and that is stable under finite intersections.

Until the end of this proof, we are going to restrict ourselves to the set of loops that surround the origin.

We say that an annular region is "good" if the origin is in the interior of its inner hole. Note that the set of loops in $\mathcal{U}_{A}$ that do surround the origin (when $A$ is not good) can be viewed as a countable unions of sets $\mathcal{U}_{A_{j}}$, where the $A_{j}$ s are good annular regions. Hence, we can restrict ourselves to the $\sigma$-field generated by good annular regions.

But the set of all $\mathcal{U}_{A}$, where $A$ 's are good annular regions, is stable under finite intersection. So, we would like to prove that the values $\nu\left(\mathcal{U}_{A}\right)$ for good annular 
regions are determined uniquely (once the constant $c$ has been chosen). Let us fix a good annular region $A$.

Conformal invariance implies that (under the previous assumptions for $\nu$ ) there exists $c>0$ such that for any two simply connected domains $V$ and $U$ with $0 \in$ $V \subset U \neq \mathbb{C}$, the $\nu$ mass of the set of loops

$$
X(V, U)=\{\gamma: \gamma \text { surrounds } 0, \gamma \not \subset V, \gamma \subset U\}
$$

is equal to $c \log \Phi^{\prime}(0)$ where $\Phi$ is the conformal map from $V$ onto $U$ that fixes the origin such that $\Phi^{\prime}(0)$ is a positive real (this follows immediately from (3.2) using the conformal equivalence of $\nu_{U}$ and $\left.\nu_{\mathbb{U}}\right)$.

We now construct another family of observables that is stable under finite intersections: We now define $U_{0}$ to be the union of $A$ with its hole. We choose $V_{0}$ to be a simply connected set containing the origin that is at positive distance from $A$. Define $X_{0}=X\left(V_{0}, U_{0}\right)$ and the family $\Pi_{0}=\Pi\left(V_{0}, U_{0}\right)$ of events $X\left(V_{0}, W\right) \subset X_{0}$ where $W$ spans all possible simply connected sets with $V_{0} \subset W \subset U_{0}$. Note that $\Pi_{0}$ is indeed stable under (finite) intersections. Formula (3.2) for a given $c$ yields the value of $\nu$ on the family $\Pi_{0}$ and its total mass on $X_{0}$, and it therefore characterizes $\nu$ on the entire $\sigma$-field $\Sigma_{0}=\Sigma\left(V_{0}, U_{0}\right)$ generated by $\Pi_{0}$.

Let us denote the outer boundary of $A$ by $a_{2}=a_{2}(A)$ and its inner boundary by $a_{1}=a_{1}(A)$. Note that $\mathcal{U}_{A} \subset X_{0}$. It is easy to check that a self-avoiding loop is in $\mathcal{U}_{A}$ if and only if, for some $m \geq 1$ and all $p \geq 1$, it intersects any (of the countably many) simple continuous path on $2^{-p} \mathbb{Z}^{2}$ that intersects $a_{2}$ and that is at distance less than $2^{-m}$ from $a_{1}$. Hence $\mathcal{U}_{A}$ is a countable union of countable intersections of sets $X\left(V_{0}, U_{0} \backslash \eta\right)$, so that $\mathcal{U}_{A} \in \Sigma_{0}$. Hence, $\nu\left(\mathcal{U}_{A}\right)$ is in fact uniquely determined.

The family $\mathcal{U}_{A}$ when $A$ spans all good annular regions is also stable under finite intersections. The value of $\nu$ on this family is determined. Furthermore the masses $\nu\left(\mathcal{U}_{A_{0}}\right)$ are finite for good annular regions $A_{0}$. It follows that for each good annulus $A_{0}, \nu$ restricted to $\mathcal{U}_{A_{0}}$ is fully determined. Since this is true for any $A_{0}$, it follows that $\nu$ itself is uniquely characterized once the constant $c$ has been chosen.

Proof of the proposition. Let us now assume that $\mu$ is a non-trivial measure on the set of self-avoiding loops in the plane that satisfies weak conformal restriction. Define $\nu$ to be its restriction to the set of loops that surround the origin. It does then satisfy the conditions of the lemma. It is therefore uniquely determined and satisfies (3.2). By translation-invariance, this characterizes $\mu$ too (because for any self-avoiding loop $\gamma$, there exists a point $z$ with rational coordinates such that $\gamma-z$ surrounds the origin), and the formula (3.1) follows from the expression of $\nu(X(V, U))$ and translation-invariance.

\section{Simply connected Case: Construction}

We have reduced the class of measures on loops that satisfy weak conformal restriction to a possibly empty one-dimensional (i.e. via multiplication by constants) family of measures. It is of course natural to try to construct such a measure, to make sure that this family is not empty. We are going to do this using the Brownian loop-measure introduced with Greg Lawler in 24.

4.1. The Brownian loop-measure. Since it is an instrumental tool throughout the present paper, it is worthwhile to recall the construction of the Brownian loopmeasure defined in [24] (in $\mathbb{C}$ and subsets of $\mathbb{C}$ ) and its basic properties. 
It is well known since Paul Lévy that planar Brownian motion is conformally invariant. This is usually stated as follows. Suppose that $Z$ is a Brownian motion started from $z \in D$ and stopped at its first exit time $T$ of the domain open $D \subset$ $\mathbb{C}$. Suppose that $\Phi$ is a conformal map from $D$ onto $\Phi(D)$. Then, up to timereparametrization, $\Phi(Z[0, T])$ is a Brownian motion started from $\Phi(z)$ and stopped at its first exit time of $\Phi(D)$.

Also, recall that $\log \left|Z_{t}\right|$ is a local martingale when $Z$ is a planar Brownian motion started away from the origin. It follows easily from these two facts that for each $z$, one can define a measure $N^{z}$ supported on Brownian loops that start and end at $z$ as follows: Consider, for each $\varepsilon>0$, the law $P_{z, \varepsilon}$ of a Brownian motion started uniformly on the circle of radius $\varepsilon$ around $z$ and stopped at its first hitting of the circle of radius $\varepsilon^{2}$. Then, consider the limit $N^{z}$ when $\varepsilon \rightarrow 0$ of

$$
4 \log (1 / \varepsilon) \times P_{z, \varepsilon} .
$$

This limit is an infinite measure supported on the set of Brownian paths $Z$ that start and end at $z$. The mass of the set of loops that reach the unit circle but not the circle of radius exp $r$ (for $r>0$ ) is $r$. Conformal invariance of planar Brownian motion implies readily that this measure $N^{z}$ is also conformally invariant in the following sense. If we consider a domain $D$ with $z \in D$ and a conformal map $\Phi: D \rightarrow \Phi(D)$, then

$$
\Phi\left(N^{z} 1_{Z \subset D}\right)=N^{\Phi(z)} 1_{Z \subset \Phi(D)} .
$$

Here, as in the rest of this paper, we do not care about the time-parametrization of the Brownian loop. We identify two Brownian loops if one can be obtained from the other by an "increasing" reparametrization (which is more than just identifying the traces since the Brownian motions have double points - as opposed to the selfavoiding loops). However, and we shall use this in a moment, a Brownian loop $Z$ carries its "natural" time-parametrization given by its quadratic variation. It has a natural time-length $T(Z)$ which is not a conformally invariant quantity. In fact, it is easy to see that

$$
T(\Phi(Z))=\int_{0}^{T(Z)}\left|\Phi^{\prime}\left(Z_{t}\right)\right|^{2} d t
$$

when $Z$ is parametrized by its "natural" time.

Now, consider the product measure $\hat{M}=d^{2} z \otimes N^{0}$, where as in the rest of this paper, $d^{2} z$ denotes the Lebesgue measure in the complex plane. This defines a couple $\left(z, Z^{0}\right)$, where $Z^{0}$ is a Brownian loop rooted at the origin and $z \in \mathbb{C}$. We then simply define $Z^{z}=z+Z^{0}$ which is now a Brownian loop rooted at $z$. Finally, we call $Z$ the equivalence class of $Z^{z}$ modulo reparametrizations (i.e. we erase the information where the root $z$ is located on $Z^{z}$ ). Then, we can view the measure $M$ defined from $\hat{M}$ by

$$
\frac{d M}{d \hat{M}}=\frac{1}{T\left(Z^{0}\right)}
$$

as a measure on the set of unrooted Brownian loops $Z$. This is the Brownian loop-measure.

Note that it is possible to conversely start with the measure $M$ on unrooted loops and to recover $\hat{M}$ by choosing a root for each loop $Z$, uniformly with respect to its time-parametrization. 


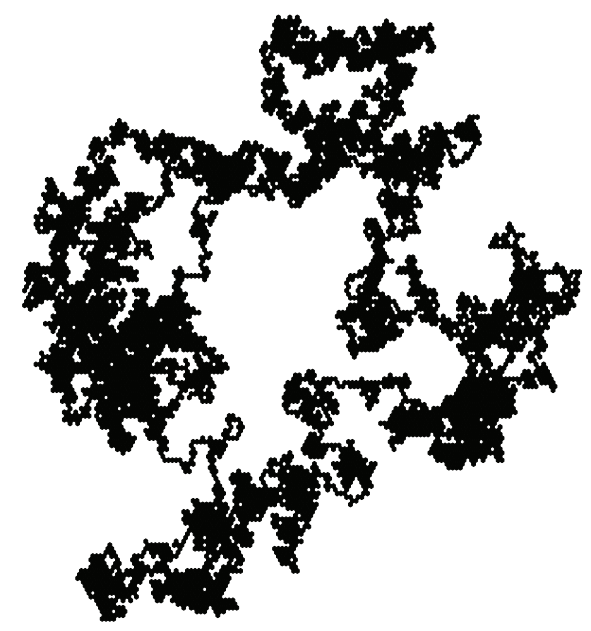

Figure 3. The trace of a (discretized) Brownian loop (its outer boundary is Figure 10

It turns out that with this definition of $M$, if we define $M_{D}=M 1_{Z \subset D}$ to be the Brownian loop-measure restricted to those loops that stay in the domain $D$, then $M$ satisfies the conformal restriction property. More precisely,

Lemma 5 (24]). For any open domain $D \subset \mathbb{C}$ and for any conformal map $\Phi$ from $D$ onto $\Phi(D)$,

$$
\Phi \circ M_{D}=M_{\Phi(D)} .
$$

This fact is derived in 24]; see also [41. It is a rather simple consequence of the conformal invariance of $N^{z}$. The idea is to start from the measure $M_{D}$ and to choose the root on a Brownian loop $Z$ with respect to another distribution other than the uniform distribution. One chooses the root $z$ according to the uniform distribution with respect to the "natural time" of $\Phi(Z)$. Then, one just has to check that the couple $(\Phi(Z), \Phi(z))$ is indeed defined under $M_{\Phi(D)}$.

Note that in this lemma, we did not require here the domain $D$ to be simply connected. However, with our present definition, we restrict ourselves to the cases where $D \subset \mathbb{C}$ (i.e. it does not have a higher genus). We shall discuss the other cases later in the paper.

4.2. Outer boundaries. Lemma 5 shows that $M$ does satisfy the same type of conformal restriction property as the one that we are looking for. But, of course, Brownian loops are not self-avoiding. However, for each Brownian loop $Z$, we can define its "outer boundary" $\partial Z$. More precisely, it is the boundary of the unbounded connected component of the complement of $Z$. It is not difficult to see that it is a continuous curve (it is the outer boundary of a continuous loop) and furthermore, it can be proved (e.g. 8]) that it is a self-avoiding loop (in other words, the Brownian loop has no cut-points - these would correspond to double cut-points on a Brownian curve, and a priori estimates can be used to prove that such points do almost surely not exist on a Brownian curve).

Hence, $M$ induces a measure on (unrooted) self-avoiding loops in the plane. This measure exists and it satisfies conformal restriction in simply connected domains, 
because the outer boundary of the image of $Z$ under a conformal map $\Phi$ defined on a simply connected domain $D$ is the image under $\Phi$ of the outer boundary of $Z$. Combining this with the results of the previous section, we get the existence of the measure $\mu$ (note that this measure can be viewed as defined on $\mathcal{G}$ because $\hat{M}$ and $M$ are a priori defined on a richer $\sigma$-field; there are no measurability problems here):

Proposition 6. There exists a non-trivial measure $\mu$ on the set of self-avoiding loops in the plane that does satisfy weak conformal restriction. It is equal (up to a multiplicative constant) to the measure on outer boundaries of Brownian loops defined via $M$.

Note that the conformal restriction property for $M$ works also for non-simply connected domains. However, this does not imply strong conformal restriction for the measure $\mu$ on self-avoiding loops; for instance, the image of the $\partial Z$ under the mapping $z \rightarrow 1 / z$ is the "inner boundary" (i.e. the boundary of the connected component that contains the origin) of $1 / Z$ and not its outer boundary.

Let us now focus on the measure $\nu$ on loops surrounding the origin described in the previous section and on its Brownian interpretation. Clearly, taking the measure $\mu$ restricted to those loops that surround the origin gives a way to construct $\nu$.

On the other hand, the measure $N^{0}$ on Brownian loops rooted at the origin defines also a measure on self-avoiding loops surrounding the origin. Furthermore, the conformal invariance property of $N^{0}$ implies that this measure on self-avoiding loops also satisfies the conformal restriction property that we require for $\nu$. Hence

Proposition 7. There exists a measure $\nu$ that satisfies the conditions of Lemma 4 , It is equal (up to a multiplicative constant) to the measure on outer boundaries of Brownian loops defined under $N^{0}$. It is also equal (up to a multiplicative constant) to the measure on outer boundaries of Brownian loops surrounding 0 defined under $M$.

Let us insist on the fact that the two Brownian measures $M 1_{\{\partial Z \text { surrounds } 0\}}$ and $N^{0}$ are very different, so that this last result can seem somewhat surprising. The second measure on loops is supported on rooted loops that go through the origin, while for the first one, (almost) no loop does go through the origin.

4.3. Consequences for Brownian loops. We now collect some further consequences of the previous facts concerning the Brownian loop-measures. These results are not really needed in our construction of the measure $\mu$ for general surfaces, but we write these consequences now to emphasize that their proof does (mostly) not use any SLE-based argument, but just properties of planar Brownian motion and conformal maps.

Proposition 7 has the following consequence:

Corollary 8. Consider two points $z$ and $\tilde{z}$ in the complex plane. The measure on outer boundaries of Brownian loops defined under $N^{z}$ and $N^{\tilde{z}}$ restricted to those loops that surround both $z$ and $\tilde{z}$ are identical.

This is just due to the fact that both measures coincide with (a constant times) the one defined by $M$ on this family of loops.

Let us remark that it is important in the previous corollary to consider the measures $N^{z}$ with no prescribed time-length for the Brownian loops. Indeed, the following fact shows that some maybe tempting conjectures are false: 
Proposition 9. Define a Brownian loop of time-length $T$ started from the point $z$ and its outer boundary $\gamma$. Define another Brownian loop of time-length T started from the point $\tilde{z}$ and its outer boundary $\tilde{\gamma}$. The laws of $\gamma$ and of $\tilde{\gamma}$ on the set of loops that surround both $z$ and $\tilde{z}$ do not (always) coincide.

Proof. One possible proof goes as follows: Consider $z=0, \tilde{z}=x$ and $T=1$, for very large real $x$. Note that loops of time-length 1 that surround both the origin and $x$ are rare. Then, we focus on the mass of the set of loops that surround the circle of radius $x$ around the origin. In the case of the Brownian loop from $x$ to $x$, a typical loop of time-length 1 with an outer boundary that surrounds the unit circle is going very fast around the circle. In the case of the loop from 0 to 0 , the loop has first to reach the circle, then to go around it, and finally to come back to the origin, and this has a much smaller probability (in the $x \rightarrow \infty$ regime).

Let us make a little general comment on shapes for scale-invariant measures on random compact sets. We are going to write it in the case of $N^{0}$, but it can clearly be adapted to the other measures that we will discuss in this paper. If we "scale out" the scale factor, we can see that $N^{0}$ defines a probability measure on the set of possible "shapes". In this Brownian case, we say that $Z$ and $Z^{\prime}$ have the same shape if for some $\lambda>0, Z=\lambda Z^{\prime}$. In other words, we look at the set of rooted loops modulo scaling.

There exist many different (measurable) ways to choose a given representative for each shape (i.e. for each equivalence class of loops). One can for instance use the "natural Brownian time-parametrization" of the loop $Z$ and choose the representative such that $T=1$ (this definition works well for $N^{0}$-almost all loops). Another possibility is to choose the representative such that the maximal distance to the origin on the loop is 1 .

Consider the probability measure $P_{0 \rightarrow 0 ; 1}$ on Brownian loops in the plane of time-length 1 (i.e. the real and imaginary parts are independent Brownian bridges of time-length 1). Define the product measure, $d r / r \otimes P_{0 \rightarrow 0 ; 1}$. If we look at $r Z$ where $(r, Z)$ is defined under this product measure, it is easy to see that it is defined under the measure $N^{0}$. Similarly, scaling shows that

$$
N^{0}=\int_{0}^{\infty} \frac{d T}{2 T} P_{0 \rightarrow 0 ; T},
$$

where $P_{0 \rightarrow 0 ; T}$ is this time the law of a Brownian loop of time-length $T$.

Clearly, for any positive real random variable $U$ (that can depend on $Z$-but does not interact with $r$ ), we see that the measure of $r U Z$ is always the same. In particular, this shows that one can view $N^{0}$ as obtained by taking the product of $r$ (defined under the measure $d r / r$ ) and a probability measure $P$ on the space of representatives (i.e. of shapes) of Brownian loops.

Conversely, one way to define $P$ from $N^{0}$ goes as follows (we safely leave this as an exercise to the reader): Define the set of loops $L_{r}$ that lie in the annulus $\{z$ : $1<|z|<r\}$. Then, consider the measure on shapes defined by $N^{0}\left(\cdot \cap L_{r}\right) / N^{0}\left(L_{r}\right)$. This is clearly a probability measure. Consider then the limit of this probability measure as $r \rightarrow \infty$. Note that $N^{0}$ and $2 N^{0}$ give rise to the same probability measure on shapes.

Hence, in a way, defining the scale-invariant measure $N^{0}$ (modulo a multiplicative constant) is equivalent to defining the probability measure $P$ on shapes of loops. 
Looking at Proposition 7, it is of course natural to ask what the multiplicative factor relating the outer boundaries defined under the measures $M$ and $N^{0}$ is and to what constants $c$ these measures correspond in the formula (3.2). In the case of $N^{0}$, things are easy: We have chosen our normalization in such a way that the $N^{0}$ mass of the set of loops with maximal radius between 1 and $e$ is 1 . Hence, it follows immediately that the constant $c$ in (3.2) is equal to 1 for $N^{0}$.

In the case of $M$, it is more complicated to compute the constant $c$. The starting point of the loop is chosen away from the origin and one has to keep only those loops that surround the origin. Let us now look at the set $A$ of self-avoiding loops that surround the origin and have time-length between 1 and $e^{2 r}$. Clearly,

$$
\begin{aligned}
M(A) & =\int_{\mathbb{C}} d^{2} z N^{z}\left(\frac{1_{A}}{T(Z)}\right) \\
& =\int_{\mathbb{C}} d^{2} z \int_{1}^{\exp (2 r)} \frac{d T}{2 T^{2}} P_{z \rightarrow z ; T}(Z \text { surrounds } 0) \\
& =\int_{\mathbb{C}} d^{2} z \int_{1}^{\exp (2 r)} \frac{d T}{2 T^{2}} P_{0 \rightarrow 0 ; T}(Z \text { surrounds } z) \\
& =\int_{1}^{\exp (2 r)} \frac{d T}{2 T^{2}} E_{0 \rightarrow 0 ; T}(\mathcal{A}(Z)) d T,
\end{aligned}
$$

where $\mathcal{A}(Z)$ denotes the area of the inside of the self-avoiding loop defined by $Z$. Clearly, $E_{z \rightarrow z ; T}(\mathcal{A}(Z))=T E_{z \rightarrow z ; 1}(\mathcal{A}(Z))$ because of scaling. Furthermore, we know from [14] that

$$
E_{z \rightarrow z ; 1}(\mathcal{A}(Z))=\pi / 5
$$

Note that this result has been obtained in [14 via considerations involving conformal restriction and SLE (this is the only time in this section where we use an SLE-based result). Hence,

$$
M(A)=\frac{\pi}{5} r .
$$

Note finally that the measure $M$ restricted to those Brownian loops that surround the origin is scale-invariant. Hence, at each scale, the area $\mathcal{A}(Z)$ scales like the time-length of $Z$. It therefore follows readily that $c$ for the case of the measure on self-avoiding loops surrounding the origin induced by $M$ is equal to $\pi / 5$, and therefore this measure is equal to $\pi / 5$ times the one defined via $N^{0}$.

In fact, it is possible to construct various other multiples of $\nu$ using the Brownian loop-measure $M$. We list some of the possibilities:

- Note that a Brownian loop $Z$ defines an integer index around any point $z \notin Z$, and in particular, around the origin (since for $M$-almost every loop $Z, 0 \notin Z$ ). Let us call $\mathcal{N}$ this index. Then, for each fixed $n \in \mathbb{Z}$, we define the measure on self-avoiding loops surrounding the origin induced by $M$, but restricted to those loops $Z$ such that $\mathcal{N}=n$. It is easy to see that this measure satisfies conformal restriction too, so that it is a multiple of the measure $N^{0}$. The constant can be easily identified via the law of the index of the Brownian loop (see [43, 14]).

- For each loop $Z$, we define the connected components $C_{j}$ of the complement of $Z$. We define a graph on this set of connected components as follows: We say that $C_{j}$ and $C_{i}$ are neighbors if there exists a point on the loop that touches both $\partial C_{i}$ and $\partial C_{j}$. It is easy to see that the existence of cut-points 
on planar Brownian curves (first proved in [7]) implies that each $C_{j}$ has infinitely many neighbors. But it is an open question whether this graph has several connected components or not. Let us call $C_{0}$ the connected component containing the origin, and $C_{\infty}$ the connected component containing infinity. For each $n \geq 1$, we can define the event $A_{n}$ that $C_{0}$ and $C_{\infty}$ are at distance exactly $n$ in the graph. If we now focus on the outer boundaries of Brownian loops restricted on the set of loops for which $A_{n}$ holds, we get also a measure satisfying conformal restriction. Hence it is also a multiple of $N^{0}$. This time, it seems difficult to compute the multiplicative constant.

At this point, it is probably worth mentioning that the Brownian loop-measure rooted at $z$ and the unrooted Brownian loop-measure defined in 24 and denoted there by $\mu(z, z)$ and $\mu^{l o o p}$ differ from $N^{z}$ and $M$ by a multiplicative constant. More precisely, $\mu(z, z)=N^{z} / 2 \pi$ and $\mu^{\text {loop }}=M / 2 \pi$. The normalization there is due to the relation between these measures and the "central charge" of the corresponding models, in the convention used in theoretical physics. In the normalization of 24, a Poissonian cloud of intensity $c \mu^{l o o p}$ (see [38]) corresponds to a representation with central charge $c$.

To conclude this section, we do not resist the temptation of citing the following visionary phrases from Mandelbrot 28]:

"a Brown loop separates the plane into two parts: an exterior which can be reached from a distant point without intersecting the loop, and an interior which I propose to call a Brown hull or Brown Island. (...) I propose for the Brown hull's boundary the term self-avoiding Brownian motion."

\section{Annular Regions}

We have now constructed a measure on self-avoiding loops as outer boundaries of planar Brownian loops. This measure satisfies weak conformal restriction and the construction of the measure is very non-symmetric: The "outside" boundary of the Brownian loop is (by definition) on the same side of the Brownian path as the boundary of the simply connected domain that we are looking at. It seems that there is little hope to say anything similar in annular regions, since the notion of inside and outside would become symmetric.

One of the main points of the present paper is however to show that - even if its Brownian construction does not work anymore - the measure on self-avoiding loops that it defines does still satisfy conformal restriction in annular regions, and therefore also on any Riemann surface.

The main statement of the present section is the following:

Proposition 10. Consider two annular regions $D$ and $\tilde{D}$ in the complex plane, such that there exists a conformal map $\Phi$ from $D$ onto $\tilde{D}$ (i.e. the two regions have the same modulus). Define $\mu_{D}$ (respectively $\mu_{\tilde{D}}$ ) as the measure $\mu$ restricted to those loops that stay in $D$ (resp. $\tilde{D})$. Then, $\Phi \circ \mu_{D}=\mu_{\tilde{D}}$.

Let us insist on the fact that in general, such a conformal map $\Phi$ does not extend conformally to the inside nor to the outside of the annular region, so that this is a much stronger conformal invariance statement than weak conformal restriction.

Note that the fact that these two measures coincide on the set of self-avoiding loops that do not go "around" the hole is not surprising and follows readily from the result in the simply connected case. However, for those loops that go around 
the hole, the result can seem surprising, and as we shall see later, it has some non-trivial consequences for Brownian loops.

In order to prove this proposition, we first need to collect and recall some relevant facts from [21, 24, 40].

In 24, we showed how to decompose a Poissonian cloud of Brownian loops in a simply connected domain, sampled according to the intensity $M$, according to the first point on a Loewner chain that the Brownian loops intersect. We are now going to recall this result, but in its "non-Poissonized" version (looking at the intensity measure instead of the Poissonian sample).

Consider the upper half-plane $\mathbb{H}$. Suppose that $\eta:[0, T] \rightarrow \overline{\mathbb{H}}$ is a continuous curve without double-points in $\mathbb{H}$, such that $\eta(0)=0, \eta(0, T] \in \mathbb{H}$. We are going to suppose that $\eta$ is parametrized in such a way that for each $t$, the conformal map $\varphi_{t}$ from $\mathbb{H} \backslash \eta(0, t]$ onto $\mathbb{H}$ such that $\varphi_{t}\left(\eta_{t}\right)=0, \eta_{t}(z) \sim z$ as $z \rightarrow \infty$ satisfies

$$
\varphi_{t}(z)=z+b+\frac{2 t}{z}+o(1 / z)
$$

as $z \rightarrow \infty$ for some real constant $b=b(t)$. For any simple continuous path $\eta$, such a parametrization clearly exists (in other words, $\eta$ is a Loewner chain, parametrized by its capacity from infinity).

We need to define the Brownian bubble measure $B u b$ in the upper half-plane: This is a measure supported on Brownian loops starting and ending at the origin but that stay in $\mathbb{H}$ in the meantime. Very roughly speaking, one can view Bub as "a version of $N^{0}$ conditioned on the loops that stay in $\mathbb{H}$ " (or a version of $M$ conditioned on the set of loops that touch the boundary of $\mathbb{H}$ just at 0 and stay in $\mathbb{H}$ otherwise). More precisely, let $Q_{\varepsilon}$ denote the law of a Brownian path started from $i \varepsilon$ and conditioned to exit the upper half-plane in the interval $[0, \varepsilon]$ (and stopped at this exit time). Note that the $Q_{\varepsilon}$ probability that the Brownian path reaches the circle of radius 1 is of the order $\varepsilon^{2}$. Indeed, the conditioning in the definition of $Q_{\varepsilon}$ is with respect to an event of constant probability (independent of $\varepsilon$ ) because of scaling. On the other hand, a Brownian motion started from $i \varepsilon$ reaches distance one from the origin without exiting the positive half-plane with probability of the order $\varepsilon$. Then, it has also a conditional probability $\varepsilon$ to exit $\mathbb{H}$ via the small interval $[0, \varepsilon]$ of size $\varepsilon$. Hence, it should not be really surprising that

$$
B u b=\lim _{\varepsilon \rightarrow 0} \varepsilon^{-2} Q_{\varepsilon}
$$

exists and that it has the following scaling property: If $Z$ is defined according to $B u b$, then $\lambda Z$ is defined according to $\lambda^{-2} B u b$. For more details, see [21, 24].

The following lemma is derived in 24] in its poissonized form.

Lemma 11 (24]). For some positive constant $c$,

$$
M_{\mathbb{H}}\left(\cdot 1_{Z \cap \eta[0, T] \neq \emptyset}\right)=c \int_{0}^{T} d t \varphi_{t}^{-1}(B u b(\cdot)) .
$$

This lemma should not be surprising: We are decomposing the measure $M_{\mathbb{H}}$ restricted to those Brownian loops that intersect $\eta$, according to the smallest $t$ such 
that $\eta_{t} \in Z$. Furthermore, intuitively speaking:

- Because of conformal restriction, the conformal Markov-type property (that leads to the definition of SLE in a slightly different context) holds, so that

$$
M_{\mathbb{H}}\left(\cdot 1_{Z \cap \eta[0, T] \neq \emptyset}\right)=\int_{0}^{T} d t \varphi_{t}^{-1}(\operatorname{Bib}(\cdot))
$$

for some measure $B i b$ supported on the set of bubbles in $\mathbb{H}$.

- This measure $B i b$ is equal to a constant times $B u b$, because when $T$ is very small, it is very close to the "conditioned" version of $M$.

We do not care here about the values of the constants as they are not needed for our purpose.

We now derive another important intermediate fact, and this is where considerations involving $\mathrm{SLE}_{8 / 3}$ come into play in a crucial way, even if it is not so apparent. The SLE-ingredients that are used are that $\mathrm{SLE}_{8 / 3}$ is a simple curve (this is proved in [31) that satisfies two-sided conformal restriction in the chordal case (this is proved in 21] and briefly recalled at the end of the introduction) :

Let us focus on the outer boundary $\gamma$ of a Brownian loop defined under the Brownian bubble measure $B u b$. We denote by $b u b$ the measure under which $\gamma$ is defined. It turns out (e.g. [21]) that one defines this measure using SLE:

Lemma 12 (21]). The measure bub is equal to the limit when $\varepsilon \rightarrow 0$ of

$$
\text { cst } \times \varepsilon^{-2} \times P_{0 \rightarrow \varepsilon}
$$

where $P_{0 \rightarrow \varepsilon}$ denotes the law of the chordal $S L E_{8 / 3}$ curve from 0 to $\varepsilon$ in the upper half-plane.

In the sequel, $P_{x \rightarrow y}$ will denote the law of chordal SLE from $x$ to $y$ in $\mathbb{H}$. Consider now a simply connected bounded closed set $A \subset \mathbb{H}$ and a conformal map $\Phi$ from the annular region $\mathbb{H} \backslash A$ onto some other annular region $\mathbb{H} \backslash \tilde{A}$ such that $\Phi(\infty)=\infty$. For convenience, we define

$$
k=k(\varepsilon, \Phi)=\left(\frac{\varepsilon^{2} \Phi^{\prime}(0) \Phi^{\prime}(\varepsilon)}{(\Phi(\varepsilon)-\Phi(0))^{2}}\right)^{5 / 8} .
$$

Lemma 13. Consider an $S L E_{8 / 3}$ curve $\beta$ from 0 to $\varepsilon$ in $\mathbb{H}$. The law of $\Phi(\beta)$, restricted to the event where $\beta \subset \mathbb{H} \backslash A$, is identical to $k_{\varepsilon}$ times the law of an $S L E_{8 / 3}$ from $\Phi(0)$ to $\Phi(\varepsilon)$ in $\mathbb{H}$, restricted to stay in $\mathbb{H} \backslash A$.

In other words,

$$
\Phi\left(1_{\beta \subset \mathbb{H} \backslash A} P_{0 \rightarrow \varepsilon}\right)=k(\varepsilon, \Phi) P_{\Phi(0) \rightarrow \Phi(\varepsilon)} 1_{\beta \subset \mathbb{H} \backslash \tilde{A}} .
$$

A related fact has been derived by Vincent Beffara in his $\mathrm{PhD}$ thesis [5], Section 5.1.2. We give here a direct justification based on $\mathrm{SLE}_{8 / 3}$ 's chordal conformal restriction property:

Proof. Consider a simple path $\delta$ that joins the real line to $A$ in $\mathbb{H}$ (and does start away from 0 and $\varepsilon$ on the real line). Note that the starting point of $\delta$ can be in the interval $(0, \varepsilon)$. Consider the image $\tilde{\delta}$ of $\delta$ under $\Phi$. Define the simply connected sets $H=\mathbb{H} \backslash(\delta \cup A)$ and $\tilde{H}=\mathbb{H} \backslash(\tilde{\delta} \cup \tilde{A})$. $\Phi$ maps conformally the former to the latter. Hence, by conformal invariance, the image of $\mathrm{SLE}_{8 / 3}$ from 0 to $\varepsilon$ in $H$ is an $\mathrm{SLE}_{8 / 3}$ from $\Phi(0)$ to $\Phi(\varepsilon)$ in $\tilde{H}$. We would like to say that the law of $1_{\beta \subset H} \Phi \circ \beta$ is identical to $k P_{\Phi(0) \rightarrow \Phi(\varepsilon)} 1_{\beta \subset \tilde{H}}$. Since by chordal restriction and conformal invariance, both 
these measures are in fact multiples of the SLE law in $\tilde{H}$, it remains only to compare the total masses.

The probability that an $\mathrm{SLE}_{8 / 3}$ from 0 to $\varepsilon$ in $\mathbb{H}$ stays in $H$ is $\left(\psi^{\prime}(0) \psi^{\prime}(\varepsilon)\right)^{5 / 8}$, where $\psi$ denotes a conformal map from $H$ onto $\mathbb{H}$ that fixes 0 and $\varepsilon$. This is $\mathrm{SLE}_{8 / 3}$ 's chordal restriction property [21].

Similarly, the probability that an $\mathrm{SLE}_{8 / 3}$ from $\Phi(0)$ to $\Phi(\varepsilon)$ in $\mathbb{H}$ stays in $\tilde{H}$ is $\left(\tilde{\psi}^{\prime}(\Phi(0)) \tilde{\psi}^{\prime}(\Phi(\varepsilon))\right)^{5 / 8}$, where $\tilde{\psi}$ is a conformal map from $\tilde{H}$ onto $\mathbb{H}$ that fixes $\Phi(0)$ and $\Phi(\varepsilon)$.

Note that $\psi^{-1} \circ \Phi \circ \psi$ maps conformally $\mathbb{H}$ onto itself and that it maps 0 to $\Phi(0)$ and $\varepsilon$ to $\Phi(\varepsilon)$. Hence, the product of its derivatives at 0 and at $\varepsilon$ is equal to $(\Phi(\varepsilon)-\Phi(0))^{2} / \varepsilon^{2}$ (because a simple scaling does the job).

Putting the pieces together, we get that for any $\delta$, the law of

$$
1_{\beta \subset \mathbb{H} \backslash(A \cup \delta)} \Phi \circ \beta
$$

is indeed identical to $k P_{\Phi(0) \rightarrow \Phi(\varepsilon)} 1_{\beta \subset \mathbb{H} \backslash(\tilde{A} \cup \tilde{\delta})}$. Since this is true for any $\delta$ (and because a simple curve from 0 to $\varepsilon$ in $H$ does not disconnect $A$ from the real axis), the lemma follows.

We now write down the consequence of the above for the bubble measure bub on self-avoiding loops in the upper half-plane:

Lemma 14. Let $A, \tilde{A}$ and $\Phi$ be as in Lemma 13. We also assume that $\Phi(0)=0$. Then, the measure $\Phi \circ\left(b u b 1_{\gamma \cap A=\emptyset}\right)$ is identical to

$$
\Phi^{\prime}(0)^{-2} b u b 1_{\gamma \cap \tilde{A}=\emptyset} .
$$

Proof. This follows readily from Lemma 12 and the properties of the SLE measure described in the last lemma. Note also of course that

$$
\lim _{\varepsilon \rightarrow 0} \frac{\varepsilon^{2} \Phi^{\prime}(0) \Phi^{\prime}(\varepsilon)}{(\Phi(\varepsilon)-\Phi(0))^{2}}=1
$$

We are now ready to prove the proposition:

Proof of Proposition 10, Consider first two annular regions of the type $\mathbb{H} \backslash A$ and $\mathbb{H} \backslash \tilde{A}$ such that there exists a conformal map $\Phi$ from the first onto the second. It suffices to prove that for any Loewner chain $\eta[0, T]$ in $\mathbb{H}$ that does not intersect $A$, the image under $\Phi$ of the measure $\mu$ restricted to those loops in $\mathbb{H}$ that do intersect $\eta$ but not $A$ is exactly identical to the measure $\mu$ restricted to those loops in $\mathbb{H}$ that do intersect $\Phi(\eta)$ but not $\tilde{A}$.

We know that

$$
1_{\gamma \cap A=\emptyset, \gamma \cap \eta[0, T] \neq \emptyset} \mu_{\mathbb{H}}=\int_{0}^{T} d t \varphi_{t}^{-1}\left(b u b 1_{\gamma \cap \varphi_{t}(A)=\emptyset}\right) .
$$

Hence, the conformal image under $\Phi$ of the left-hand side of the previous expression is equal to

$$
\int_{0}^{T} d t \Phi \circ \varphi_{t}^{-1}\left(b u b(\cdot) 1_{\left.\gamma \cap \varphi_{t}(A)=\emptyset\right)} .\right.
$$

The path $\Phi(\eta[0, T])$ is also a Loewner chain. But its parametrization by capacity is different from that of $\eta$. Let $s=s(t)$ denote the capacity at infinity of $\Phi(\eta[0, t])$ in $\mathbb{H}$, and let us define $\tilde{\eta}$ by $\tilde{\eta}_{s(t)}=\Phi\left(\eta_{t}\right)$, so that $\tilde{\eta}$ is a naturally parametrized 


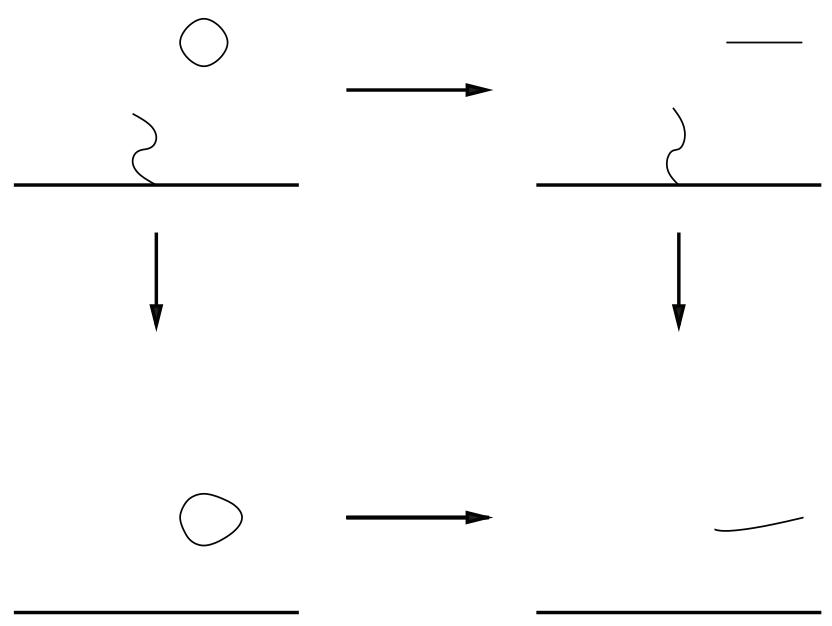

Figure 4 . The commutative diagram with $\Phi, \Phi_{t}, \varphi_{t}$ and $\tilde{\varphi}_{s}$

Loewner chain. Let us define the conformal map $\tilde{\varphi}_{s}$ from $\mathbb{H} \backslash \tilde{\eta}[0, s]$ onto $\mathbb{H}$, such that $\tilde{\varphi}_{s}(z) \sim z$ at infinity and $\tilde{\varphi}_{s}\left(\tilde{\eta}_{s}\right)=0$.

We now define $A_{t}=\varphi_{t}(A), \tilde{A}_{s}=\tilde{\varphi}_{s}(\tilde{A})$, and $\Phi_{t}$, the conformal map from $\mathbb{H} \backslash A_{t}$ onto $\mathbb{H} \backslash \tilde{A}_{s(t)}$ defined by

$$
\Phi_{t}=\tilde{\varphi}_{s(t)} \circ \Phi \circ \varphi_{t}^{-1}
$$

It is normalized at infinity and maps the origin to the origin. Note that

$$
s(t)=\int_{0}^{t} d u\left|\Phi_{u}^{\prime}(0)\right|^{2}
$$

for scaling reasons: The capacity of $\varphi_{t}(\eta[t, t+\varepsilon])$ is $\varepsilon$ by definition. The capacity of $\tilde{\varphi}_{s(t)}(\tilde{\eta}[s(t), s(t+\varepsilon)])$ is $s(t+\varepsilon)-s(t)$ by definition. But the second slit is the image of the first one by $\Phi_{t}$, and it is then standard that $s(t+\varepsilon)-s(t) \sim \varepsilon\left|\Phi_{t}^{\prime}\left(\eta_{t}\right)\right|^{2}$ when $\varepsilon \rightarrow 0$.

Hence, if $S=s(T)$,

$$
\begin{aligned}
\Phi & \circ\left(1_{\gamma \cap A=\emptyset, \gamma \cap \eta[0, T] \neq \emptyset} \mu_{\mathbb{H}}\right) \\
& =\int_{0}^{S} d s\left|\Phi_{t}^{\prime}(0)\right|^{-2} \tilde{\varphi}_{s}^{-1} \circ \Phi_{t}\left(b u b(\cdot) 1_{\gamma \cap \varphi_{t}(A)=\emptyset}\right) \\
& =\int_{0}^{S} d s \tilde{\varphi}_{s}^{-1} \circ b u b(\cdot) 1_{\gamma \cap \tilde{A}_{s}=\emptyset} \\
& =1_{\gamma \cap \tilde{A}=\emptyset, \gamma \cap \tilde{\eta}[0, S] \neq \emptyset} \mu_{\mathbb{H}} .
\end{aligned}
$$

But this is true for any Loewner chain $\eta[0, T]$ in $\mathbb{H} \backslash A$. Hence, it follows that

$$
\Phi\left(1_{\gamma \cap A=\emptyset} \mu_{\mathbb{H}}\right)=1_{\gamma \cap \tilde{A}=\emptyset} \mu_{\mathbb{H}} .
$$

This is exactly the statement of the proposition in the case where $D=\mathbb{H} \backslash A$ and $\tilde{D}=\mathbb{H} \backslash \tilde{A}$.

In the general case (i.e. general annular domains $D$ and $\tilde{D}$ ), define $D_{1}$ (resp. $\tilde{D}_{1}$ ) to be the complement of the unbounded connected component of $\mathbb{C} \backslash D$ (resp. $\mathbb{C} \backslash \tilde{D})$, i.e. the domain obtained by filling the hole of $D$ (resp. $\tilde{D})$. One can map 
conformally $D_{1}$ (resp. $\tilde{D}_{1}$ ) onto the upper half-plane and apply the result to the images of $D$ (resp. $\tilde{D}$ ) under these maps (these are sets of the type $\mathbb{H} \backslash A$ and $\mathbb{H} \backslash \tilde{A}$ as before). Then, mapping things back onto $D$ and $\tilde{D}$, we get immediately the proposition for $D$ and $\tilde{D}$.

\section{General Riemann surfaces}

We can now turn our attention to general Riemann surfaces. In the sequel, we suppose that $\mu$ is defined in such a way that the constant $c$ defined in (3.2) is equal to one.

6.1. Definition of $\mu$ and conformal restriction. Let $\rho(D)$ denote the modulus of an annular region $D$, i.e. the number such that there exists a conformal map from $D$ onto the annulus $\{z: 1 \leq|z|<\exp (\rho)\}$. We say that a self-avoiding loop in $D$ goes around the hole in $D$ if it disconnects the inner part of $\partial D$ from its outer part (in $D$ ). Then

Lemma 15. There exists a function $F:(0, \infty) \rightarrow(0, \infty)$ such that for any annular region $D \subset \mathbb{C}$,

$$
\mu(\{\gamma: \gamma \subset D \text { and goes around the hole in } D\})=F(\rho(D)) .
$$

This is just due to the fact that $\mu$ satisfies conformal invariance for annular regions. $S$ :

This will allow us to justify the following definition of $\mu_{S}$ for any Riemann surface

Definition. Let us now consider any Riemann surface $S$. We say that $D \subset S$ is an annular region if it is conformally equivalent to an annulus. Then, we define $\mu_{S}$ by the fact that for any annular region $D \subset S$,

$$
\mu_{S}(\{\gamma: \gamma \subset D \text { and goes around the hole in } D\})=F(\rho(D)),
$$

where $F$ is the function defined in the previous lemma.

Let us first show that this definition indeed determines $\mu_{S}$ uniquely: The family of events of the type

$$
A_{D}=\{\gamma: \gamma \subset D \text { and goes around the hole in } D\}
$$

when $D$ varies in the family of annular regions in $S$ is stable under finite intersections. It is also easy to check that this family of events generates the $\sigma$-field on which $\mu_{S}$ is defined. Hence, standard measure-theoric considerations imply that there exists at most one measure $\mu_{S}$ such that $\mu_{S}\left(A_{D}\right)=F(\rho(D))$ for any annular region $D \subset S$.

Let us now check that the measure $\mu_{S}$ exists. Each self-avoiding loop in $S$ is contained in some annular domain $D \subset S$ (consider for instance a "small neighborhood" of the loop). One can in fact easily define a countable family of annular domains $D_{n} \subset S$ such that each loop is contained in at least one domain $D_{n}$. We have defined for each $D_{n}$ a measure $\mu_{D_{n}}$ on loops in the annular region $D_{n}$ in the previous section. These families are compatible since $\mu_{D_{n}}$ and $\mu_{D_{m}}$ do coincide on $D_{n} \cap D_{m}$ (the measure does satisfy restriction in annular regions). Hence, this indeed constructs a measure $\mu_{S}$ on loops in $S$, and for any $n, \mu_{S} 1_{\gamma \subset D_{n}}=\mu_{D_{n}}$. 
The conformal restriction property of the family of measures $\mu_{S}$ then follows immediately from the conformal restriction property in the annular regions derived in the previous section.

We have now in fact derived all the ingredients needed to check Theorem 2

Conclusion of the proof of Theorem 2, We have just constructed a family $\left(\mu_{S}\right)$ that satisfies conformal restriction. It remains to check that it is the unique one (up to a multiplicative constant).

To prove this, we can note that the previous argument shows that the whole family $\left(\mu_{S}\right)$ is characterized by the subfamily $\left(\mu_{D}\right)$ where $D$ spans the family of annular regions. Hence, the whole family is in fact characterized by $\mu=\mu_{\mathbb{C}}$ because one can then define all $\mu_{D}$ 's for annular regions by restriction. But we have seen that there exists a unique measure $\mu$ (up to a multiplicative constant) on loops in $\mathbb{C}$ that satisfies conformal restriction for simply connected domains. Hence, the family $\left(\mu_{S}\right)$ is unique too.

6.2. Relation to Brownian loops, zero genus. Let us recall the steps in our construction of $\mu$. In the simply connected case (and for genus $g=0$ ), we constructed the measure $\mu$ using outer boundaries of Brownian loops defined under the loop-measure. Then, using the relation with $\mathrm{SLE}_{8 / 3}$, we proved that this measure $\mu$ satisfies an inside/outside symmetry property, and this enabled us to define the measure $\mu$ on any Riemann surface. This raises naturally the question of the relation between the measure $\mu$ on a surface $S$ and measures on Brownian loops on $S$.

Let us first focus on the case where the genus of $S$ is zero, i.e. $S=D$ is a subset of the Riemann sphere. In this case, the Brownian loop-measure in $D$ is simply $M_{D}$, i.e. the restriction of the measure $M$ on Brownian loops in the plane to the set of loops that stay in $D$.

If one considers a bounded annular region $D \subset \mathbb{C}$ such that the origin is in the hole, we can consider the conformal map $z \mapsto 1 / z$ defined from $D$ onto the annular region $1 / D$. The image of $\mu_{D}$ is exactly $\mu_{1 / D}$. Hence, the image of the self-avoiding curve $1 / \gamma$ can be viewed as the outside boundary of a Brownian loop $Z^{\prime}$. But because of the invariance of the Brownian loop-measure $M$ under the map $z \mapsto 1 / z$ (this is due to its conformal invariance), we see that we can also view $1 / \gamma$ as the "inside boundary" of a Brownian loop $1 / Z$. In fact, we have just proved the following:

Proposition 16. Consider a Brownian loop $Z$ defined under the Brownian loopmeasure $M$, and call its outer boundary $\gamma$ as before. Define also the boundary $\bar{\gamma}$ of the connected component of $\mathbb{C} \backslash Z$ that contains the origin. Note that $\gamma=\bar{\gamma}$ unless $Z$ disconnects 0 from infinity. Then, the measure under which $\bar{\gamma}$ is defined is identical to the measure under which $\gamma$ is defined (and is equal to $\mu$ ).

Suppose that $Z$ is a Brownian loop defined under the measure $M$. Define the family $\left(\gamma_{j}\right)$ of its boundaries (i.e. the boundaries of the connected components of its complement. Then, define the measure on self-avoiding loops $\hat{\mu}$ as

$$
\hat{\mu}(A)=M\left(\sum_{j} 1_{\gamma_{j} \in A}\right)
$$




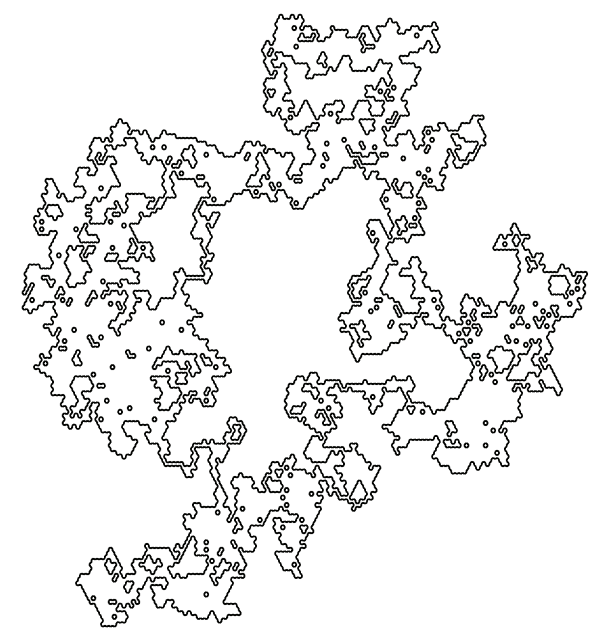

Figure 5. The boundaries in the Brownian loop from Fig. 3

(i.e. we take all loops simultaneously). Note that the complement of the Brownian loop has of course only finitely many large connected components (the number of small loops has been studied in [29, 25]).

Corollary 17. We have $\hat{\mu}=2 \mu$.

Proof. The measure $\hat{\mu}$ is clearly translation-invariant. Furthermore, if we look at the measure $\hat{\mu}$ restricted to those loops that disconnect the origin from infinity, we see that we count once the inner boundary and once the outer boundary (if $Z$ disconnects the origin) and nothing else. The result follows.

For any multiply connected subset $D$ of the plane, it is always possible to view $\mu_{D}$ as a measure on outer boundaries of Brownian loops: One just has to fill in all the holes except a given prescribed one (this defines a simply connected subset of the Riemann sphere) and to consider outer boundaries of Brownian loops defined in this domain.

The conformal invariance of $\mu_{D}$ has some other consequences. For instance, consider $D_{1}=\mathbb{C} \backslash \mathbb{U}$ and $D_{2}=\mathbb{C} \backslash[-1,1]$. These two sets are conformally equivalent (let $\psi$ denote the conformal map from $D_{2}$ onto $D_{1}$ that is symmetric with respect to the real axis). One can view $\mu_{D_{1}}$ as the measure on outer boundaries $\gamma$ of Brownian loops $Z$ in $\mathbb{C}$, restricted to those loops such that $\gamma \cap \mathbb{U}=\emptyset$. But, one can also view it as the conformal image via $\psi$ of the measure of outer boundaries of Brownian loops $Z$ in $\mathbb{C}$ such that $\gamma \cap[-1,1]=\emptyset$. Hence, as far as outer boundaries that do not intersect $\mathbb{U}$ are concerned, one can replace the Brownian loops in $\mathbb{C}$ by "Brownian loops in the simply connected domain obtained from $\mathbb{C} \backslash \mathbb{U}$ by identifying all conjugated points $e^{i \theta}$ and $e^{-i \theta}$ on $\partial \mathbb{U} . "$

Let us also note that the fact that one Brownian loop defines infinitely many boundaries is not in contradiction to the fact that $\hat{\mu}=2 \mu$ because we are talking about scale-invariant (infinite) measure. In fact, since the Lebesgue measure of a Brownian path is zero, it is clear that the area enclosed by the outer boundary of a Brownian loop is identical to the sum of all areas of the inner boundaries of 
the same loop (so that this sum converges), which is another way to see why this identity holds (with the factor 2).

Also, recall that for a Brownian loop of time-length 1, it is known [29, 25] that the number of inner boundaries that enclose an area at least $u$ behaves almost surely like $2 \pi /\left(u\left(\log (1 / u)^{2}\right)\right)$ as $u \rightarrow \infty$ (this question was also raised by Mandelbrot). Furthermore, the result of 36 shows that in fact it is enough to look at the sample of one Brownian loop to describe fully the measure $\mu$ in $\mathbb{C}$ : If $F$ is a function of the "shape" (i.e. a function invariant under scaling and translations) of a connected component and if $C_{1}, C_{2}, \ldots$ are the bounded connected components of the complement of the Brownian loop ordered by decreasing area, then [36 $\left(F\left(C_{1}\right)+\ldots+F\left(C_{n}\right)\right) / n$ tends (in $\left.L^{2}\right)$ to " $P(F)$ ", where $P(F)$ is in fact the expected value of $F$ for the probability measure on the shape of the outer boundary of a Brownian loop.

6.3. Relation to Brownian loops: Higher genus. Let us now say a few words on the relation between Brownian loop-measures and $\mu_{S}$, when the surface $S$ has a higher genus (for instance when $S$ is the torus $\mathbb{C} /(\mathbb{Z}+\tau \mathbb{Z})$ ).

We can start with the case where $S$ is compact (since the other cases will follow from restriction). We first have to define properly the Brownian loop-measure. It is quite clear how to proceed: For each $z \in S$, we can define the Brownian loopmeasure $N_{S}^{z}$ in $S$ rooted at $z$ in a similar way as in $\mathbb{C}$ by taking the limit when $\varepsilon \rightarrow 0$ of $4 \log (1 / \varepsilon)$ times the law of a Brownian motion on $S$, started uniformly on the circle of radius $\varepsilon$ around $z$ and stopped at its first hitting time of the circle of radius $\varepsilon^{2}$ around $z$. Note that we can define for each loop, its natural time $T(Z)$ (given for by its total quadratic variation), but that this notion depends on the choice of a metric on $S$ (they are only defined locally modulo conformal invariance).

After arguing that the map $z \rightarrow N^{z}$ is in some sense measurable (one can for instance couple $N^{z}$ and $N^{z^{\prime}}$ when $z$ and $z^{\prime}$ are close), we can define the measure

$$
\hat{M}_{S}=\int_{S} d^{2} z N^{z} \frac{1}{T(Z)}
$$

where $d^{2} z$ denote here the chosen metric on $S$. This is now a measure on the set of rooted Brownian loops in $S$. Finally, we erase the information of where the root is on $Z$ and define the corresponding measure $M_{S}$ on unrooted Brownian loops in $S$. Note that when the genus of $S$ is zero, this is the same as the Brownian measure that we have studied earlier in this paper. The proof of conformal invariance and restriction for this family of measures $M_{S}$ is then essentially identical to that of the restrictions of the planar measure $M_{\mathbb{C}}$.

A first remark is that the boundaries of connected components of the complement of a Brownian loop defined under $M_{S}$ are not necessarily self-avoiding anymore, even for "planar connected components". Consider for instance the torus $S=\mathbb{C} /(\mathbb{Z}+i \mathbb{Z})$. It can happen (i.e. with positive mass) that the Brownian loop goes around the torus in both directions (i.e. for instance that in the covering surface $\mathbb{C}$ it goes through the neighborhood of the origin, then stays close to the segment $[0,1]$ and then to the segment $[1,1+i]$ and closes the loop near $1+i=0)$ and that the path has local cut-points. At such a cut-point, locally, both sides of the Brownian motion are in the same connected component of $S \backslash Z$. Hence, the boundary of this connected component has a double point (and in fact, it has many cut-points, i.e. the dimension of the set of cut-points is $3 / 4$; see [18]). 
On the other hand, for a given surface $S$, the $M_{S}$-mass of the set of loops such that the boundaries of the connected components of its complement in $S$ are not all simple curves is finite. Hence, focusing our attention on those loops that have simple boundaries is not so restrictive. One could then see whether there is a direct relation to the measure $\mu$.

The answer does not seem a priori obvious. Recall for instance from [39] that a Brownian excursion "conditioned to have no cut-point" defines a restriction measure of parameter 2, i.e. the outer boundary is the same as two $\mathrm{SLE}_{8 / 3}$ 's conditioned not to intersect. This shows that there might (maybe surprisingly) still exist a direct relation between $\mu$ and boundaries of some Brownian loops in this case.

The measure $\mu_{S}$ defines (deterministic) global quantities related to the Riemann surface $S$. For instance, one can look at the total mass of the set of loops in a given homology class. These quantities therefore provide ways to describe the moduli space of Riemann surfaces. Furthermore, the restriction property of $\mu$ makes it wellsuited to the study of "local deformations" of the complex structure. In particular, suppose that two Riemann surfaces $S$ and $S^{\prime}$ coincide almost everywhere but are different "inside a small circle $C$ ". Then, the only difference between $\mu_{S}$ and $\mu_{S^{\prime}}$ concerns just those loops that go through the domain encircled by $C$. Hence, one can control the variation of the macroscopic observables defined by the measures $\mu$ with respect to "infinitesimal variations" of the Riemann structure. We plan to investigate this further in upcoming work [42].

Recent attention (e.g. 44, 4]) has been devoted to the question of generalizing the definition of SLE to non-simply connected domains. More generally, one can try to define SLE on any Riemann surface. The present construction of $\mu$ on any Riemann surface together with the construction of conformal loop-ensembles [38, 34] (see also [4]) via Poissonian clouds of self-avoiding loops gives a way to define families of SLE-loops on any Riemann surface (even if it does not have a boundary). Note that in the case of surfaces with boundaries (and therefore finite Green's functions), a Poissonian cloud of Brownian loops leads to the same type of simple CLE, but for compact surfaces, the Brownian loop-clusters become dense and uninteresting, while the clusters of self-avoiding loops still make sense.

\section{Asymptotics for the FUnCtion $F$}

7.1. Motivation. A motivation for the present paper is the desire to understand better the behavior of long self-avoiding walks on planar lattices and the related conjectures. For some background and recent status on these conjectures, see [20]. Let us mention here the following conjecture from [20]: Consider a regular periodic planar lattice $L$ (to fix ideas, let us suppose that the lattice is the square lattice, the triangular lattice or the hexagonal lattice). Recall that there exists a latticedependent constant $\lambda>1$ such that the number of self-avoiding paths of length $n$ on $L$ that start at the origin behaves like $\lambda^{n+o(1)}$ when $n \rightarrow \infty$.

Let us now define the discrete measure $\mu^{\delta}$ on the set of self-avoiding loops that one can draw on $\delta L$, that (for each $n$ ) puts a weight $n^{-\lambda}$ to each loop with $n$ steps.

Conjecture 1 ([20]). $\mu^{\delta}$ converges when $\delta \rightarrow 0$ to a limiting measure on selfavoiding loops.

If this limiting measure is conformally invariant, then it is easy to see that it must be (a multiple of) our measure $\mu$. So, if one believes in this conjecture and 
in conformal invariance in the scaling limit, then the fact that the definition of $\mu$ makes sense on any Riemann surface and satisfies restriction in a broad sense should not be surprising.

One may wonder if a further study of the (conjectural) limiting measure $\mu$ might be of some help to understand the conjecture better. This is what motivates the next subsection.

7.2. Asymptotics. It would be nice to be able to have an explicit expression for the mass of the set of loops that go around an annulus in terms of its modulus (i.e. the explicit expression of $F$ ). This does not seem easy. We are now going to study the asymptotics of $F$ when $\rho \rightarrow 0$.

Proposition 18. For some constant $c_{0}, F(\rho) \sim c_{0} e^{-5 \pi^{2} / 4 \rho}$ when $\rho \rightarrow 0$.

In other words, if one considers a very thin tube of width 1 around a very long smooth curve of length $l$, the mass of the set of loops that go around the tube decays exponentially in $l$. This is of course reminiscent of the fact that for the discrete grid-model $\mu^{\delta}$, the mass decays exponentially in the number of steps.

As we shall see, the exponent $5 \pi^{2} / 4$ is very closely related to the $5 / 8$ exponent for $\mathrm{SLE}_{8 / 3}$ as derived in [21.

Before proving this fact, let us first concentrate on the corresponding result for the bubble measure. For convenience, we will first use the unit disc rather than the upper half-plane. Define $f(\rho)$ (up to a multiplicative constant) as the limit as $\varepsilon \rightarrow 0$ of $\varepsilon^{-2}$ times the probability that an $\mathrm{SLE}_{8 / 3}$ from 1 to $\exp (i \varepsilon)$ in $\mathbb{U}$ goes "around" (i.e. clockwise in this case) the disc $e^{-\rho} \mathbb{U}$ without hitting it. We want to control this probability uniformly with respect to $\rho$ when $\varepsilon \rightarrow 0$.

Lemma 19. For some positive constant $c, f(\rho) \sim c \rho^{-2} \exp \left(-5 \pi^{2} /(4 \rho)\right)$ when $\rho \rightarrow$ 0 .

Proof of the lemma. It is convenient to use the Poissonian excursion representation of $\mathrm{SLE}_{8 / 3}$ introduced in [40]. Let us briefly recall how it goes in the present case of the unit disc. First, for each $\theta \in[0,2 \pi)$, we define the Brownian excursion measure $\mathcal{E}^{\theta}$ at $e^{i \theta}$ in $\mathbb{U}$ as the limit when $\delta \rightarrow 0$ of $\delta^{-1} \times P_{\exp (-\delta+i \theta)}^{B M}$, where $P_{z}^{B M}$ denotes the law of a Brownian motion started from $z$ and stopped at its first exit of $\mathbb{U}$. Then, define the excursion measure $\mathcal{E}=\int_{0}^{2 \pi} d \theta \mathcal{E}^{\theta}$. This is an infinite measure, supported on the set of Brownian paths in $\mathbb{U}$ that start and end on $\partial \mathbb{U}$ (each excursion has a starting point and an endpoint). It has been first defined in 23] and for instance used in 24, 40. It possesses strong conformal invariance properties (see e.g. 24]).

Then, take two points $u_{1}$ and $u_{2}$ on $\partial \mathbb{U}$, and let $\partial_{1}, \partial_{2}$ denote the two connected components of $\partial \mathbb{U} \backslash\left\{u_{1}, u_{2}\right\}$. Consider a Poissonian realization of $c \mathcal{E}$ restricted to those excursions that have both their endpoints on $\partial_{1}$, where $c$ is a well-chosen constant. Consider the curve from $u_{1}$ to $u_{2}$ in $\mathbb{U}$ that delimits the boundary of the connected component (that contains $\partial_{2}$ as the other part of its boundary) of the complement in $\mathbb{U}$ of the union of all these excursions in $\mathbb{U}$. Then (see 40]), for this well-chosen definition of $c$, the curve $\eta$ is exactly an $\mathrm{SLE}_{8 / 3}$ from $u_{1}$ to $u_{2}$.

In the case where $u_{1}=1$ and $u_{2}=e^{i \varepsilon}$ and $\partial_{1}$ is the "long arc" $\left\{e^{i \theta}, \theta \in[\varepsilon, 2 \pi]\right\}$, then clearly, if we want $\eta$ to go around the disc $e^{-\rho} \mathbb{U}$, necessarily, none of the excursions is allowed to hit $e^{-\rho} \mathbb{U}$.

The $\mathcal{E}^{\theta}$ mass of the set of excursions that hit $e^{-\rho} \mathbb{U}$ is equal to $1 / \rho$ (just because $\log |Z|$ is harmonic for a planar Brownian motion $Z$ ). Hence, the $\mathcal{E}$-mass of the 
set of excursions that hit $e^{-\rho \mathbb{U}}$ is equal to $2 \pi / \rho$. Also, the $\mathcal{E}$-mass of the set of excursions that hit $e^{-\rho} \mathbb{U}$ and has their starting point (resp. endpoint) in $\partial_{1}$ is $(2 \pi-\varepsilon) / \rho$. Hence, the $\mathcal{E}$-mass of the set of excursions that hit $e^{-\rho} \mathbb{U}$ and have both their endpoints in $\partial_{1}$ is greater than $(2 \pi-2 \varepsilon) / \rho$. It follows that the probability $p_{\varepsilon, \rho}$ that none of the excursions used to construct the SLE from $u_{1}$ to $u_{2}$ in $\mathbb{U}$ does hit $e^{-\rho} \mathbb{U}$ satisfies

$$
\exp \{-2 \pi c / \rho\} \leq p_{\varepsilon, \rho} \leq \exp \{-2(\pi-\varepsilon) c / \rho\}
$$

(recall that for a Poissonian sample of intensity measure $m$, the probability that in the Poissonian sample no item belongs to the set $A$ is $\exp \{-m(A)\})$.

We say that an excursion in the annulus $\mathbb{U} \backslash e^{-\rho} \mathbb{U}$ goes the easy way if it disconnects 1 from $e^{-\rho} \mathbb{U}$ in $\mathbb{U}$. Otherwise, we say that it goes the "hard way". When no excursions intersect the disc $e^{-\rho \mathbb{U}}$, then the SLE itself does not intersect the smaller disc either. It then remains to estimate the (conditional) probability that the SLE goes "the hard way" (i.e. "around $\left.e^{-\rho} \mathbb{U} "\right)$. This means exactly that none of the following two events happens:

- There exists a Brownian excursion that goes the easy way.

- There exist two Brownian excursions that do not go the easy way, such that their union disconnects 1 from $e^{-\delta} \mathbb{U}$ in $\mathbb{U}$.

We know already that this conditional probability decays like $g(\rho) \varepsilon^{2}$ when $\rho$ is fixed and $\varepsilon \rightarrow 0$ for some positive $g(\rho)$ because of the convergence of the renormalized SLE measure to the bubble measure (i.e. $g(\rho) \exp (-2 \pi c / \rho)$ is the mass for the bubble measure of the set of bubbles that go around $\left.e^{-\rho} \mathbb{U}\right)$.

On the other hand, it is easy to note that because of scaling, when $\varepsilon \rightarrow 0$, the probabilities of this event for $\varepsilon, \delta$ and the event for $2 \varepsilon, 2 \delta$ become very close (for instance by comparing this event with the event in the strip $\mathbb{R} \times[0, \delta])$ ). Hence, we get that $g(\rho)=c s t / \rho^{2}$.

We have now proved that for some constants $c_{1}, c_{2}$,

$$
f(\rho) \sim c_{1} \rho^{-2} \exp \left\{-c_{2} / \rho\right\}
$$

when $\rho \rightarrow 0$. In fact, it is possible to identify what the constant $c_{2}$ is because we know that the chordal restriction exponent of $\mathrm{SLE}_{8 / 3}$ is $5 / 8$ (see [21]). In particular, this enables us for instance to estimate the probability that an SLE from -1 to 1 in the unit disc stays in $\mathbb{U} \backslash\left(e^{-\rho} \mathbb{U} \cup[-i, 0]\right)$ and the leading order term when $\rho \rightarrow 0$ can be interpreted in terms of Brownian excursions in $\mathbb{U}$. The upshot is that $c_{2}=5 \pi^{2} / 4$. Intuitively, going around the annulus is at first order like crossing the rectangle $[0,2 \pi] \times[0, \rho]$. By scaling, this is like crossing the rectangle $\left[0, L=2 \pi^{2} / \rho\right] \times[0, \pi]$, and for an $\mathrm{SLE}_{8 / 3}$, this has probability of order $\exp (-5 L / 8)$.

Proof of the proposition. It now remains to deduce the result for the loop-measure $\mu$ from the result on bubbles. For this, we are going to use the decomposition of $\mu$ along a Loewner chain. Suppose that $\eta:[0, T] \rightarrow \overline{\mathbb{U}}$ now denotes a continuous simple path in the unit disc $\mathbb{U}$, started from $\eta_{0}=1$. We asume that it is parametrized from "capacity" at the origin as radial Loewner chains usually are, i.e. that if we define the conformal maps $\varphi_{t}$ from $U_{t}=\mathbb{U} \backslash \eta[0, t]$ onto $\mathbb{U}$ such that $\varphi_{t}(0)=0$ and $\varphi\left(\eta_{t}\right)=1$, then $\left|\varphi_{t}^{\prime}(0)\right|=\exp t$. In fact, we may want to think of $\eta=\eta^{\varepsilon}$ as a spiral that winds very fast around the origin (say a time-reparametrization of $\exp (-t+i t / \varepsilon)$ for a very small $\varepsilon$ ). Then, for small $\varepsilon$, the domain $U_{t}$ is (conformally speaking) very close to the disc $e^{-t} \mathbb{U}$. 
The function $f(r)$ can be viewed as the bubb-measure of the set of bubbles that go around the disc $e^{-\rho}$, where $b u b b$ is "the bubble measure in the unit disc", started at the boundary point 1 . Exactly as in the case of the upper half-plane, one can prove that

Hence, it follows that

$$
\mu_{\mathbb{U}} 1_{\gamma \cap \eta[0, T]}=\int_{0}^{T} d t \varphi_{t}^{-1} \circ b u b b .
$$

$$
\mu_{\mathbb{U}}\left(\gamma \cap \eta[0, T] \neq \emptyset \text { and } \gamma \text { surrounds } e^{-\rho} \mathbb{U}\right)=\int_{0}^{T} f(r(t)) d t
$$

where $r(t)=\rho\left(\mathbb{U} \backslash\left(\eta[0, t] \cup e^{-\rho} \mathbb{U}\right)\right)$. If we take $T=\rho$, and $\eta=\eta^{\varepsilon}$ as before, and then let $\varepsilon \rightarrow 0$, we get immediately that

$$
F(\rho)=\mu_{\mathbb{U}}\left(\gamma \text { surrounds } e^{-\rho} \mathbb{U}\right)=\int_{0}^{\rho} f(r) d r .
$$

In particular, when $\rho \rightarrow 0$,

$$
F(\rho) \sim c_{1} \exp \left(-5 \pi^{2} / 4 \rho\right)
$$

\section{Relation to CRIticAl PERCOLATiON}

We are going to conclude this paper by briefly mentioning in a more informal style the direct relation between $\mu$ and the measures on outer perimeters of critical percolation clusters in their scaling limit. This will show that, as in the case of Brownian loops, the inside perimeters and the outside perimeters of critical percolation clusters have the same "law" and also that outside perimeters are invariant (in law) with respect to perturbations of the complex structure "inside".

Let us consider critical percolation on the triangular lattice in the entire plane (we restrict ourselves to this lattice since it is at present the only one for which conformal invariance is proved). We can view it as a random coloring of the faces of a hexagonal grid, where each face is colored independently in black or white with probability $1 / 2$. One way to encode the obtained configuration is to define the family of loops on the hexagonal grid that separate white connected components and black connected components. These are the interfaces for this model. Each of the loops is self-avoiding on the scale of the lattice and the family of all loops describes fully the percolation configuration.

For convenience, we are going to introduce a small $\delta$ and to look at the percolation configuration on a grid of mesh-size $\delta$ (we shall then let $\delta \rightarrow 0$ ). It is easy to see using the Russo-Seymour-Welsh theory that for any bounded domain $D$ and any $\varepsilon>0$, the mean number of loops included in $D$ and of diameter greater than $\varepsilon$ remains bounded and bounded away from 0 (except of course if $\varepsilon$ is greater than the diameter of $D)$ as $\delta \rightarrow 0$.

Furthermore, it is also easy to see that the loops will "not remain self-avoiding" on the large scale, i.e. that on a large loop, one can find neighboring sites $x$ and $x^{\prime}$ on the hexagonal lattice of mesh-size $\delta$ and on the same loop such that the loop goes (twice) to a macroscopic distance between its visits of $x$ and $x^{\prime}$.

Smirnov 35. proved that in the scaling limit crossing probabilities of conformal rectangles for this percolation model converge to a (conformally invariant) limit predicted by Cardy [10] when $\delta$ goes to zero. Camia and Newman [9] showed, using 


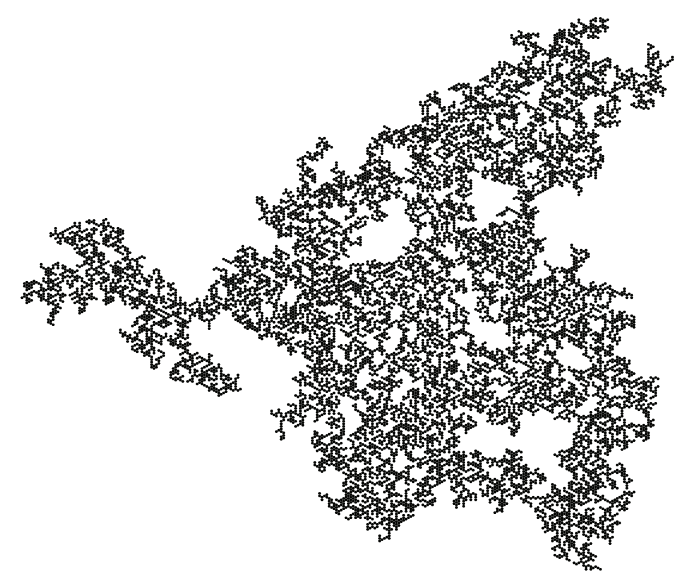

Figure 6. A percolation cluster

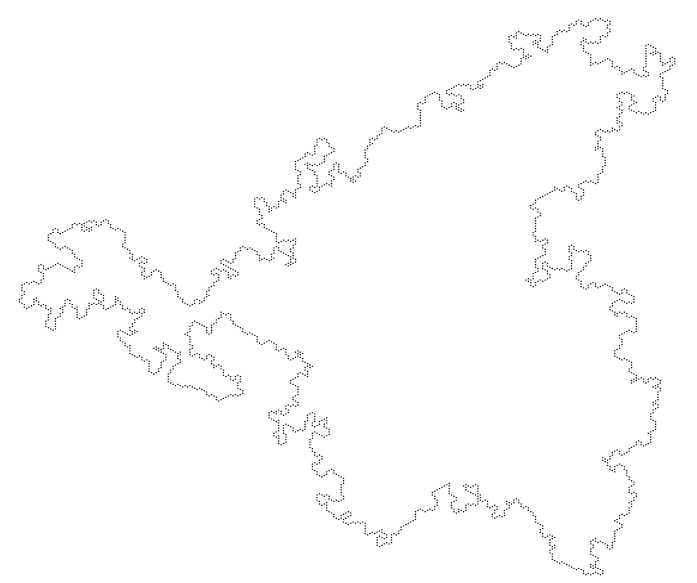

Figure 7. Its outer loop

Smirnov's result, how one can indeed deduce convergence when $\delta \rightarrow 0$ of the joint law of all the discrete loops, towards a continuous family of loops, that are also described in terms of the $\mathrm{SLE}_{6}$ processes that had been studied in [17, 18. This continuous family of loops in some sense describe the joint law of the clusters in the scaling limit. In particular, one loop out of two is the outer boundary of a white cluster, the other ones being "inside boundaries" of white clusters. Let us call $\left(o_{j}, j \in J\right)$ these outer boundaries.

These continuous loops are not self-avoiding (in fact, they have many double points: the dimension of each loop is a.s. $7 / 4$ while the dimension of their "boundaries" is $4 / 3$; see e.g. [6]). Let us call $O_{j}$ the self-avoiding loop obtained by taking the "outer boundary" (i.e. the boundary of the unbounded connected component of the complement) of $o_{j}$. The conformal invariance statement can then be phrased 
as follows:

For each simply connected domain $D$, define the joint law $P_{D}$ of the set of loops $\left(o_{j}, j \in J_{D}\right)$ that do stay in $D$. Then, if $\Phi$ is a conformal map from $D$ onto another simply connected domain $\tilde{D}$, then the law of $\left(\Phi\left(o_{j}\right), j \in J_{D}\right)$ is identical to $P_{\tilde{D}}$.

Clearly, this implies also the same statement for the outer boundaries $O_{j}$ instead of the boundaries $o_{j}$. Hence, the probability measures $P_{D}$ satisfy a type of conformal restriction property. In fact, if we now define for each measurable set $A$ of selfavoiding loops

$$
\pi(A)=E\left(\#\left\{j \in J: O_{j} \in A\right\}\right),
$$

then $\pi$ is a measure on the set of self-avoiding loops in the plane, that does satisfy conformal restriction. Hence, it is equal to a constant times the measure $\mu$ that we have defined in this paper. In other words, the measure $\mu$ can also be constructed as the outer boundary of the scaling limits of percolation cluster boundaries. The multiplicative constant $c$ does here not seem to be easy to determine.

Note that in fact, as we are focusing only on these outer boundaries $O_{j}$, we do not really need the convergence of the full discrete loops to the full loops $o_{j}$. Indeed, it is not very hard using some a priori estimates for percolation (i.e. the 5 -arm estimate) to see that the $O_{j}$ 's are the limits when $\delta \rightarrow 0$ of the discrete outer perimeters of percolation clusters, where the outer perimeter of a cluster is the outer boundary of the domain obtained by "filling in all fjords of width one". More precisely, consider the unbounded connected component of the complement of a cluster. We then just keep the subdomain of this component consisting of points $x$ such that there exist two disjoint paths from $x$ to infinity that avoid the cluster. The boundary of this subdomain is the discrete outer perimeter (these discrete curves had been studied and simulated by physicists before; see e.g. [1] and the references therein). Then, the law of the joint family of discrete outer perimeters $\left(O_{j}^{\delta}, j \in J^{\delta}\right)$ converges when $\delta \rightarrow 0$ to that of $\left(O_{j}, j \in J\right)$, so that for some constant $c$ and any (reasonable) set of (macroscopic but bounded) loops (for instance the set of loops that go around the hole in a given conformal annulus),

$$
\mu(A)=c \lim _{\delta \rightarrow 0} E\left(\#\left\{j \in J^{\delta}: O_{j}^{\delta} \in A\right\}\right) .
$$

The shape of a percolation cluster outer perimeter has therefore exactly the same law as that of the outer boundary of a Brownian loop. Note again that the proof of this result relies solely on the conformal invariance of both models and on their restriction property.

The definition of the discrete outer perimeter is very non-symmetric, and it is non-local in the discrete case: Because of the "closure of fjords procedure", it does not suffice to look at the status of the hexagons that touch a given self-avoiding loop to decide if it is an outer perimeter. One needs to make sure that the "fjords do not meet in the inside". Hence, the inside/outside symmetry statement for $\mu$ is not obvious at all from this perspective either (and this statement is based on SLE considerations). In fact, it has rather surprising consequences, just as for the Brownian loop approach. It says that outer perimeters of percolation are (in law) not affected by change of the conformal structure in their inside.

As for the Brownian measure $M$ in Corollary 17, one can also characterize $\mu$ in terms of all perimeters (not only the outer ones) and this changes the constant $c$ by a factor of 2 . We safely leave this to the reader. It is not difficult to check that the joint law (i.e. measure) of the boundaries for one percolation cluster is 


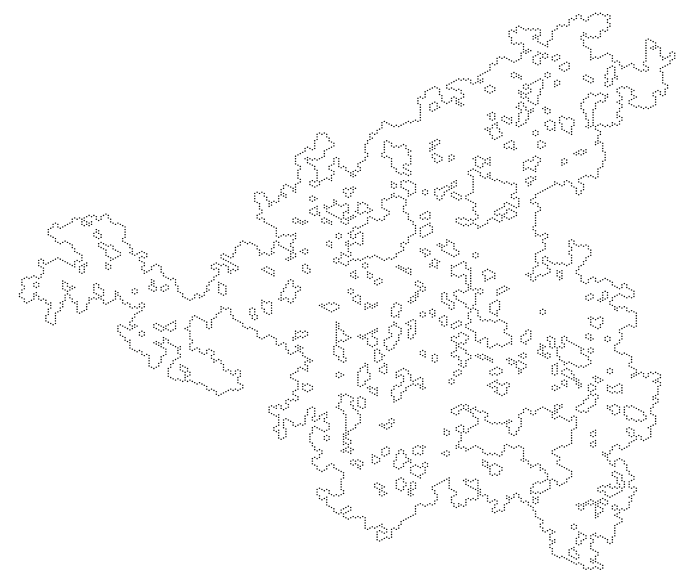

FiguRE 8. Its outer and inner loops

not the same as the joint law (i.e. measure) of the boundaries for one Brownian loop. For instance, the existence of cut-points for Brownian motions shows that in a Brownian loop, there exist inner boundaries that intersect the outer boundary and that the dimension of these intersection points is a.s. 3/4 (see [18]). For the scaling limit of percolation clusters, this is not the case (it would correspond to a "6-arms" exponent - recall that we are looking at outer boundaries). It is just the shape of "one prescribed" boundary that coincides for percolation clusters and for Brownian loops.

Finally, note that in the case of Riemann surfaces of higher genus, the cut-point issue raised in the Brownian loop case (i.e. the fact that the outer boundary can have double points) does not occur in the case of outer boundaries of percolation clusters, because the perimeters' sides have a definite "color".

\section{ACKNOWLEDGEMENTS}

I would like to thank Pierre Nolin for the pictures of the Brownian loop, the percolation cluster and their boundaries.

\section{REFERENCES}

[1] M. Aizenman, B. Duplantier, A. Aharony (1999), Connectivity Exponents and External Perimeter in 2D Independent Percolation Models, Phys. Rev. Lett. 83, 1359.

[2] R. Bass, Probabilistic techniques in analysis, Springer, 1994. MR1329542 (96e:60001)

[3] M. Bauer, D. Bernard (2004), SLE martingales and the Virasoro algebra, Phys. Lett. B 557, 309. MR:1972482 (2004k:82074)

[4] R. Bauer, R. Friedrich (2005), On chordal and bilateral SLE in multiply connected domains, preprint.

[5] V. Beffara (2000), Mouvement Brownien plan, SLE, invariance conforme et dimensions fractales, Thèse de Doctorat, Université Paris-Sud.

[6] V. Beffara (2004), Hausdorff dimensions for $\mathrm{SLE}_{6}$, Ann. Probab. 32, 2606-2629. MR 2078552 (2005k:60295)

[7] K. Burdzy (1989), Cut points on Brownian paths, Ann. Probab. 17, 1012-1036. MR.1009442 (90m:60091)

[8] K. Burdzy, G.F. Lawler (1990), Non-intersection exponents for random walk and Brownian motion II. Estimates and application to a random fractal, Ann. Prob. 18, 981-1009. MR:1062056 (91g:60097) 
[9] F. Camia, C. Newman (2005), The Full Scaling Limit of Two-Dimensional Critical Percolation, preprint.

[10] J.L. Cardy (1984), Conformal invariance and surface critical behavior, Nucl. Phys. B 240, 514-532.

[11] R. Friedrich, On connections of Conformal Field Theory and Stochastic Loewner Evolutions, math-ph/0410029.

[12] R. Friedrich, J. Kalkkinen (2004), On Conformal Field Theory and Stochastic Loewner Evolution, Nucl. Phys. B687, 279-302. MR2059141 (2005b:81173)

[13] R. Friedrich, W. Werner (2003), Conformal restriction, highest-weight representations and SLE, Comm. Math. Phys. 243, 105-122. MR2020222 (2005b:81172)

[14] C. Garban, J.A. Trujillo-Ferreras (2005), The expected area of the Brownian loop is $\pi / 5$, Comm. Math. Phys., to appear.

[15] M. Kontsevich (2003), CFT, SLE and phase boundaries, Preprint of the Max Planck Institute (Arbeitstagung 2003), 2003-60a.

[16] G.F. Lawler (2005), Conformally invariant processes in the plane, Math. surveys and monographs, AMS. MR2129588 (2006i:60003)

[17] G.F. Lawler, O. Schramm, W. Werner (2001), Values of Brownian intersection exponents I: Half-plane exponents, Acta Mathematica 187, 237-273. MR1879850 (2002m:60159a)

[18] G.F. Lawler, O. Schramm, W. Werner (2001), Values of Brownian intersection exponents II: Plane exponents, Acta Mathematica 187, 275-308. MR1879851(2002m:60159b)

[19] G.F. Lawler, O. Schramm, W. Werner (2001), The dimension of the Brownian frontier is 4/3, Math. Res. Lett. 8, 401-411 MR1849257(2003a:60127b)

[20] G.F. Lawler, O. Schramm, W. Werner (2004), On the scaling limit of planar self-avoiding walks, in Fractal Geometry and applications, a jubilee of Benoît Mandelbrot, Proc. Symp. Pure Math. 72, vol. II, AMS, 339-364. MR2112127 (2006d:82033)

[21] G.F. Lawler, O. Schramm, W. Werner (2003), Conformal restriction properties. The chordal case, J. Amer. Math. Soc., 16, 917-955. MR1992830 (2004g:60130)

[22] G.F. Lawler, J.A. Trujillo-Ferreras (2007), Random walk loop-soup, Trans. A.M.S. 359 767787. MR2255196

[23] G.F. Lawler, W. Werner (2000), Universality for conformally invariant intersection exponents, J. Europ. Math. Soc. 2, 291-328. MR1796962 (2002g:60123)

[24] G.F. Lawler, W. Werner (2004), The Brownian loop-soup, Probab. Th. Rel. Fields 128, 565-588. MR2045953 (2005f:60176)

[25] J.F. Le Gall (1991), On the connected components of the complement of a two-dimensional Brownian path, in Prog. Probab. 28, Birhauser, Boston, 323-338. MR:1146456 (93d:60132)

[26] J.F. Le Gall (1992), Some properties of planar Brownian motion, Cours de l'école d'été de St-Flour 1990, P.L. Hennequin ed., L.N. Math. 1527, 111-235. MR.1229519 (94g:60156)

[27] P. Malliavin (1999), The canonic diffusion above the diffeomorphism group of the circle, C. R. Acad. Sci. Paris I, 329, 325. MR 1713340 (2000e:60129)

[28] B.B. Mandelbrot, The Fractal Geometry of Nature, Freeman, 1982. MR665254 (84h:00021)

[29] T. Mountford (1989), On the asymptotic number of small components created by planar Brownian motion, Stoch. Stoch. Rep. 28, 177-188 MR1020270 (91b:60065)

[30] A.A. Kirillov, D.V. Yuriev (1987), Kähler geometry on the infinite dimensional manifold $\operatorname{Diff}\left(S^{1}\right) / \operatorname{Rot}\left(S^{1}\right)$, Funct. Anal. Appl. 21, 35-46. MR 925071 (89c:22032)

[31] S. Rohde, O. Schramm (2005), Basic properties of SLE, Ann. Math. 161, 879-920. MR2153402 (2006f:60093)

[32] O. Schramm (2000), Scaling limits of loop-erased random walks and uniform spanning trees, Israel J. Math. 118, 221-288. MR1776084 (2001m:60227)

[33] O. Schramm, S. Sheffield (2005), in preparation.

[34] S. Sheffield, W. Werner (2005), in preparation.

[35] S. Smirnov (2001), Critical percolation in the plane: conformal invariance, Cardy's formula, scaling limits, C. R. Acad. Sci. Paris S. I Math. 333, 239-244. MR1851632 (2002f:60193)

[36] W. Werner (1994), Sur la forme des composantes connexes du complémentaire de la courbe brownienne plane, Probab. Th. Rel. Fields 98, 307-337. MR.1262969 (95b:60101)

[37] W. Werner (2004), Random planar curves and Schramm-Loewner Evolutions, in 2002 StFlour summer school, L.N. Math. 1840, 107-195. MR2079672 (2005m:60020)

[38] W. Werner (2003), SLEs as boundaries of clusters of Brownian loops, C. R. Acad. Sci. Paris, Ser. I Math. 337, 481-486. MR.2023758 (2005b:60221) 
[39] W. Werner (2004), Girsanov's theorem for $\operatorname{SLE}(\kappa, \rho)$ processes, intersection exponents and hiding exponents, Ann. Fac. Sci. Toulouse 13, 121-147. MR2060031 (2005b:60262)

[40] W. Werner (2005), Conformal restriction and related questions, Probability Surveys 2, 145190. MR2178043

[41] W. Werner (2005), Some recent aspects of random conformally invariant systems, Lecture Notes from Les Houches summer school, July 2005.

[42] W. Werner, in preparation.

[43] M. Yor (1980), Loi de l'indice du lacet brownien et distribution de Hartman-Watson, Z. Wahrsch. Verw. Gebiete 53, 71-95. MR576898 (82a:60120)

[44] D. Zhan (2004), Random Loewner chains in Riemann surfaces, PhD dissertation, Caltech.

Université Paris-Sud, Laboratoire de Mathématiques, Université Paris-Sud, BÂt. 425, 91405 Orsay cedex, France and DMA, Ecole Normale Supérieure, 45 rue d’Ulm, 75230 PARIS CEDEX, France

E-mail address: wendelin.werner@math.u-psud.fr 